Journal for ImmunoTherapy of Cancer

\title{
Inhibition of integrin $\alpha v \beta 6$ sparks T-cell antitumor response and enhances immune checkpoint blockade therapy in colorectal cancer
}

Philipp Busenhart (1) , ${ }^{1}$ Ana Montalban-Arques, ${ }^{1}$ Egle Katkeviciute, ${ }^{1}$ Yasser Morsy, ${ }^{1}$ Chiara Van Passen, ${ }^{2}$ Larissa Hering, ${ }^{1}$ Kirstin Atrott, ${ }^{1}$ Silvia Lang, ${ }^{1}$ Jesus Francisco Glaus Garzon, ${ }^{3}$ Elisabeth Naschberger, ${ }^{2}$ Arndt Hartmann, ${ }^{4}$ Gerhard Rogler, ${ }^{1}$ Michael Stürzl, ${ }^{2}$ Marianne Rebecca Spalinger, ${ }^{1}$ Michael Scharl ${ }^{1}$

\section{ABSTRACT}

To cite: Busenhart $P$,

Montalban-Arques A, Katkeviciute $\mathrm{E}$, et al. Inhibition of integrin $\alpha v \beta 6$ sparks T-cell antitumor response and enhances immune checkpoint blockade therapy in colorectal cancer. Journal for ImmunoTherapy of Cancer 2022;10:e003465. doi:10.1136/ jitc-2021-003465

- Additional supplemental material is published online only. To view, please visit the journal online (http://dx.doi.org/10. 1136/jitc-2021-003465).

Accepted 31 December 2021

Check for updates

(C) Author(s) (or their employer(s)) 2022. Re-use permitted under CC BY-NC. No commercial re-use. See rights and permissions. Published by BMJ.

For numbered affiliations see end of article.

Correspondence to Professor Michael Scharl; michael.scharl@usz.ch cancer.
Background Integrin $\alpha v \beta 6$ is a heterodimeric cell surface protein whose cellular expression is determined by the availability of the integrin $\beta 6$ subunit (ITGB6). It is expressed at very low levels in most organs during tissue homeostasis but shows highly upregulated expression during the process of tumorigenesis in many cancers of epithelial origin. Notably, enhanced expression of integrin $\alpha v \beta 6$ is associated with aggressive disease and poor prognosis in numerous carcinoma entities. Integrin $\alpha v \beta 6$ is one of the major physiological activators of transforming growth factor- $\beta$ (TGF- $\beta$ ), which has been shown to inhibit the antitumor T-cell response and cause resistance to immunotherapy in mouse models of colorectal and mammary cancer. In this study, we investigated the effect of ITGB6 expression and antibody-mediated integrin $\alpha \mathrm{v} \beta 6$ inhibition on the tumor immune response in colorectal

Methods Using orthotopic and heterotopic tumor cell injection, we assessed the effect of ITGB6 on tumor growth and tumor immune response in wild type mice, mice with defective TGF- $\beta$ signaling, and mice treated with antiintegrin $\alpha v \beta 6$ antibodies. To examine the effect of ITGB6 in human colorectal cancer, we analyzed RNAseq data from the colon adenocarcinoma dataset of The Cancer Genome Atlas (TCGA-COAD).

Results We demonstrate that expression of ITGB6 is an immune evasion strategy in colorectal cancer, causing inhibition of the antitumor immune response and resistance to immune checkpoint blockade therapy by activating latent TGF- $\beta$. Antibody-mediated inhibition of integrin $\alpha v \beta 6$ sparked a potent cytotoxic T-cell response and overcame resistance to programmed cell death protein 1 (PD-1) blockade therapy in ITGB6 expressing tumors, provoking a drastic increase in anti-PD-1 treatment efficacy. Further, we show that the majority of tumors in patients with colorectal cancer express sufficient ITGB6 to provoke inhibition of the cytotoxic T-cell response, indicating that most patients could benefit from integrin $\alpha v \beta 6$ blockade therapy.

Conclusions These findings propose inhibition of integrin $\alpha v \beta 6$ as a promising new therapy for colorectal cancer, which blocks tumor-promoting TGF- $\beta$ activation, prevents tumor exclusion of cytotoxic T-cells and enhances the efficacy of immune checkpoint blockade therapy.

\section{BACKGROUND}

The cell surface receptor integrin $\alpha v \beta 6$ is expressed exclusively in epithelial cells. While its expression is minimal in most healthy epithelia, it is highly upregulated during carcinogenesis of many epithelial cancers. ${ }^{1-3}$ Expression of integrin $\alpha v \beta 6$ correlates with decreased survival in numerous carcinomas, such as colorectal cancer (CRC), ${ }^{45}$ breast cancer, ${ }^{6}$ pancreatic ductal adenocarcinoma ${ }^{7}$ non-small cell lung cancer, ${ }^{8}$ cervical squamous cell carcinoma ${ }^{9}$ and others. ${ }^{12}$ Integrins are heterodimeric transmembrane receptors consisting of $\alpha$ and $\beta$ subunits. The ratelimiting factor for the formation of the $\alpha v \beta 6$ heterodimer is the availability of the subunit integrin $\beta 6$ (ITGB6), which can only form a receptor complex together with the $\alpha \mathrm{v}$ integrin subunit (ITGAV). ${ }^{10}$

A key function of integrin $\alpha v \beta 6$ is the activation of transforming growth factor- $\beta$ (TGF- $\beta) .^{2}$ In fact, integrin $\alpha v \beta 6$, together with integrin $\alpha_{v} \beta 8$, are the major activators of TGF- $\beta$ in vivo. ${ }^{11}$ TGF- $\beta$ is sequestered in the extracellular matrix (ECM) as a latent compound, which is formed by TGF- $\beta$, latency-associated peptide (LAP) and latent TGF- $\beta$ binding proteins, forming the large latent complex (LLG). The concentration of TGF- $\beta$ stored within the ECM in its latent form is several orders of magnitude higher than required to produce potent biological effects. Thus, TGF- $\beta$ signaling is predominantly regulated by activation of latentTGF $\beta .^{11}$ Within the TGF- $\beta$ family, integrin $\alpha v \beta 6$ activates TGF- $\beta 1$, the most abundant and most commonly dysregulated isoform of 
TGF- $\beta$ in cancer, and TGF- $\beta 3 .{ }^{11}$ TGF- $\beta$ activation through integrin $\alpha v \beta 6$ occurs by binding of the RGD (ArgGly-Asp) peptide present in LAP that causes a conformational change in the LLC, which leads to the release of TGF- $\beta$ from the LLC. ${ }^{121011}$ Activated TGF- $\beta$ can then bind to TGF- $\beta$ type 2 receptor (TGFBR2), which recruits, transphosphorylates and activates TGF- $\beta$ type 1 receptor (TGFBR1). Activated TGFBR1 phosphorylates the transcription factors SMAD2 and SMAD3, which subsequently translocate into the nucleus and modulate the expression of target genes together with other transcription cofactors. ${ }^{11-13}$

TGF- $\beta$ signaling inhibits T-cell proliferation and effector functions as well as T-cell differentiation into the Th1 subtype, which mediates prominent and wellcharacterized $\mathrm{T}$ cell responses against cancers. Additionally, TGF- $\beta$ directly inhibits the cytotoxic program of CD8+ Tcells by repressing the expression of several genes involved in their lytic function, such as perforin, granzyme $\mathrm{A}$ and $\mathrm{B}$, and interferon-gamma (IFN- $\gamma) .{ }^{12}{ }^{13}$ In mouse models of colorectal and breast cancer, TGF- $\beta$ inhibition promotes infiltration of T-cells into the tumor and causes a potent cytotoxic T-cell response against tumor cells. ${ }^{1415}$ Further, tumors that are resistant to antiPD-L1 immune checkpoint blockade therapy (CBT) are rendered susceptible by combining this treatment with TGF- $\beta$ inhibition. This combination treatment provokes a vigorous antitumor immunity and tumor regression. ${ }^{14} 15$ A recent study showed that selective inhibition of TGF- $\beta 1$ activation is sufficient to overcome CBT resistance and to induce a profound antitumor response when combined with anti-programmed cell death protein 1 (PD-1) treatment. ${ }^{16}$ However, TGF- $\beta$ signaling is involved in many physiological processes; thus, suppression of this pathway may lead to harmful off-target effects. Continuous longterm blockade of TGF- $\beta 1$ signaling causes hemorrhagic lesions within the heart valves, as well as aortic aneurysms in rats and dogs. ${ }^{17-19}$ The promising results of targeting TGF- $\beta$ in preclinical models are therefore contrasted by modest success in clinical trials, where it proved difficult to determine a safe and effective dose. ${ }^{20}$ A more promising approach to inhibit TGF- $\beta$ signaling in a clinical setting might therefore be the prevention of TGF- $\beta$ activation by blocking integrin $\alpha \mathrm{v} \beta 6$. Since integrin $\alpha \mathrm{v} \beta 6$ is predominantly expressed during carcinogenesis, ${ }^{12}$ harmful offtarget effects might be greatly reduced compared with direct and systemic TGF- $\beta$ inhibition.

TGF- $\beta$ activation through integrin $\alpha v \beta 6$ has been shown to affect T-cell function in triple-negative breast cancer (TNBC) and in the gastrointestinal tract. In TNBC, integrin $\alpha v \beta 6$ activates TGF- $\beta$, which upregulates one of its target genes SOX4 in the tumor cells, causing resistance to $\mathrm{T}$ cell-mediated cytotoxicity. ${ }^{21}$ In the small intestine, integrin $\alpha v \beta 6$ regulates the retention of tissueresident memory T-cells by activating TGF- $\beta .^{22}$ These findings prompted us to investigate whether integrin $\alpha v \beta 6$ is affecting the T-cell immune response in CRC.
We show that ITGB6 inhibits the T-cell antitumor response and strongly accelerates tumor growth in murine models of CRC. Moreover, anti-ITGB6 treatment rendered tumors susceptible to PD-1 blockade, which caused a potent immune reaction and tumor shrinkage. Finally, we demonstrate that ITGB6 inhibits the T-cell immune response in the majority of human CRC tumors. These findings suggest upregulation of ITGB6 as an immune evasion strategy of tumor cells leading to the reduced survival rates seen in patients with tumors expressing ITGB6. Combining CBTs with anti-ITGB6 treatment might therefore be a promising, safe approach to overcome immune evasion and to increase the treatment success of current immunotherapies in CRC.

\section{METHODS \\ Study design}

Appropriate group sizes were determined on the basis of our previous experience with the models. In general, experiments aimed to include five mice per group, which were randomly assigned to the different treatments. Endpoint was reached when tumors were over 1000 $\mathrm{mm}^{3}$ or $1 \mathrm{~cm}$ in length or when mice showed wounds at the site of injection or poor health. These animals were euthanized and excluded from the analysis. Treatments were randomly performed and cage locations randomly assigned during the course of the experiments. Investigators administering the treatments were not blinded. Investigators terminating the experiments and processing the samples were blinded. No data outliers were excluded.

\section{Mice}

C57BL/6JRj, BALB/c and BALB/c nude mice were purchased from Janvier Labs (France). CD4-dnTGFBR2 (stock number 005551) and C57BL/6J (control for CD4dnTGFBR2) were purchased from The Jackson Laboratory (USA). All mice were kept in specific-pathogen-free conditions. C57BL/6JRj, BALB/c and $\mathrm{BALB} / \mathrm{c}$ nude mice were females between 9 and 11 weeks old at the start of the experiment. CD4-dnTGFBR2 and C57BL/6J were 9 weeks old at the start of the experiment.

\section{Cloning of expression vector and viral transduction}

For the construction of the ITGB6 overexpression vector, ITGB6 gene was amplified from ITGB6 cDNA ORF Clone in Cloning Vector (Sino Biological: MG50097-M) and cloned into the expression vector pLenti CMV GFP Blast (659-1) (Addgene plasmid \# 17445) ${ }^{23}$ in place of GFP. Cloning, lentiviral transduction, western blot and co-immunoprecipitation (Co-IP) were performed according to standard procedures as described in the supplementary methods.

\section{Tumor models and treatments}

Tumor cells were suspended in cell culture medium mixed 1:1 with matrigel (Corning 354263) and injected into the cecum wall (orthotopic model) or subcutaneously 
into the flanks of the mice (heterotopic model) as described in detail in the supplementary methods. In the othotopic model, mice were euthanized $\sim 2$ weeks after injection. In the subcutaneous model, tumor development was measured three times per week using a digital caliper. Tumor volume was calculated using the ellipsoid formula: $4 / 3 * 3.14 *$ Length $/ 2 *$ (Width $/ 2$ ), ${ }^{2}$ where the shorter dimension was used as width and depth. Mice were terminated 14 days after subcutaneous injection. RT-qPCR analysis and histological staining and analysis of the tumor tissue were performed according to standard procedures as described in the supplementary methods.

$\alpha v \beta 6$ blockade was performed by injecting $4 \mathrm{mg} / \mathrm{kg}$ anti- $\alpha v \beta 6$ antibody (Biogen; 6.8G6 or Biogen; 6.3G9) ${ }^{24}$ i.p. three times per week. All mice within one experiment received either anti- $\alpha v \beta 6$ antibody or the same amount of IgG isotype control antibody (Biogen; 1E6). PD-1 blockade was performed by injecting $10 \mathrm{mg} / \mathrm{kg}$ antiPD1 (CD279) antibody (BioXCell; clone 29F.1A12) i.p. three times per week. All mice within one experiment received either anti-PD1 antibody or the same amount of IgG isotype control antibody (BioXCell; clone 2A3). 5-Fluorouracil (5-FU) was administered by i.p. injections of $50 \mathrm{mg} / \mathrm{kg} 5$-FU dissolved in dimethyl sulfoxide (DMSO) at days 6, 9, and 12 after tumor cell injection. All mice within one experiment received either 5-FU treatment or the same amount of DMSO as vehicle control.

\section{Flow cytometry}

Spleen, lymph node (LN) and tumor cells were used for flow cytometry analysis. Single cell suspensions from spleen and LN were prepared as described previously. ${ }^{25}$ Cecum and subcutaneous tumors were cut to approximately $0.5 \mathrm{~mm}^{3}$ pieces and digested in $6 \mathrm{~mL}$ RPMI medium containing $0.5 \mathrm{mg} / \mathrm{mL}$ collagenase type IV (Sigma Aldrich) and $0.05 \mathrm{mg} / \mathrm{mL}$ DNAse I (Roche) solution for $10 \mathrm{~min}$ on a shaker $(300 \mathrm{rpm})$ at $37^{\circ} \mathrm{C}$. Cells were homogenized by passing the digested samples through a $18 \mathrm{G} 1.5$ syringe and centrifuged for $10 \mathrm{~min}, 4^{\circ} \mathrm{C}, 1500 \mathrm{rpm}$. Single cell suspensions were stained and restimulated as described previously. ${ }^{26} \mathrm{~A}$ list of the used antibodies is given in online supplemental table 1.

\section{RNAseq and data analysis}

Subcutaneous MC38-ITGB6 and MC38-Ctrl tumors were used for RNAseq analysis. RNA isolation and sequencing were performed by Microsynth AG, Balgach, Switzerland. We used FastQC (V.0.11.5) to check the data quality, and mouse genome mm10 from Ensemble as a reference genome for STAR (V.2.5.4) for mapping the reads. The DESeq2 R package (V.1.28.1) ${ }^{27}$ was used for detecting differentially expressed genes (DEGs). The negative binomial model and Wald test were performed together with Benjamini-Hochberg for multiple comparisons correction (false discovery rate (FDR) cut-off $<0.05$ ). A principal component analysis was performed across all the samples using the count of reads per kilobase per million mapped reads (RPKM). A heatmap was generated from unsupervised clustering using DEGs. We used EnhancedVolcano R package (V.1.8.0) for the visualization of DEGs by volcano plot. Gene ontology (GO) was carried out using Database for Annotation, Visualization, and Integrated Discovery tool V.6.8. ${ }^{28}{ }^{29}$ We used quanTIseq software ${ }^{30}$ to quantify different immune cell types. The deconvolution was based on the expression signature panel provided by the software. To summarize the signatures of T-TBRS and F-TBRS, Z-scores were computed for each gene and sample, averaged across all genes included in the profile. Signature differences between groups were assessed using Mann-Whitney tests.

\section{Human sample collection and expression analysis}

Expression analysis was conducted on tumor specimen of a prospectively collected cohort of patients with colon carcinoma who underwent surgery at the University Medical Center Erlangen. Clinical documentation was externally monitored in full. Exclusion criteria included preoperative radiation or chemotherapy, patients suffering from hereditary CRC (familial adenomatous polyposis, hereditary non-polyposis CRC) or inflammatory bowel disease (Crohn's disease, ulcerative colitis). RT-qPCR analysis and Immunohistochemical (IHC) staining of the human tumor specimen was performed as described in the supplementary methods.

\section{Human RNAseq data analysis}

The human gene expression dataset and the corresponding clinical data were retrieved from Genomic data commons data portal https://portal.gdc.cancer.gov/. The colon adenocarcinoma dataset from The Cancer Genome Atlas (TCGA-COAD) contains data from 521 patients with CRC grouped according to the tumor stage. The default trimmed mean of M-values implemented in EdgeR package (V.3.26.8) ${ }^{31} 32$ was used for gene counts normalization.

\section{Statistics}

When comparing two groups, unpaired two-tailed t-test was used. For human RNAseq data, Mann-Whitney test was used. For comparisons between three or more groups, one-way analysis of variance (ANOVA) was used and Tukey's post-hoc tests applied. Tumor growth curves were analyzed by two-way ANOVA and Tukey's post-hoc test. Statistics for DEG analysis of RNAseq are detailed above.

\section{RESULTS}

\section{ITGB6 promotes tumor growth by inhibiting the T-cell antitumor immune response}

To investigate the effect of ITGB6 on tumor development, we overexpressed ITGB6 in the two murine CRC cell lines CT26 and MC38, which present with low endogenous ITGB6 expression (figure 1A). ITGB6 overexpression was performed by viral transduction with an ITGB6-expressing vector (ITGB6) and control cells were 

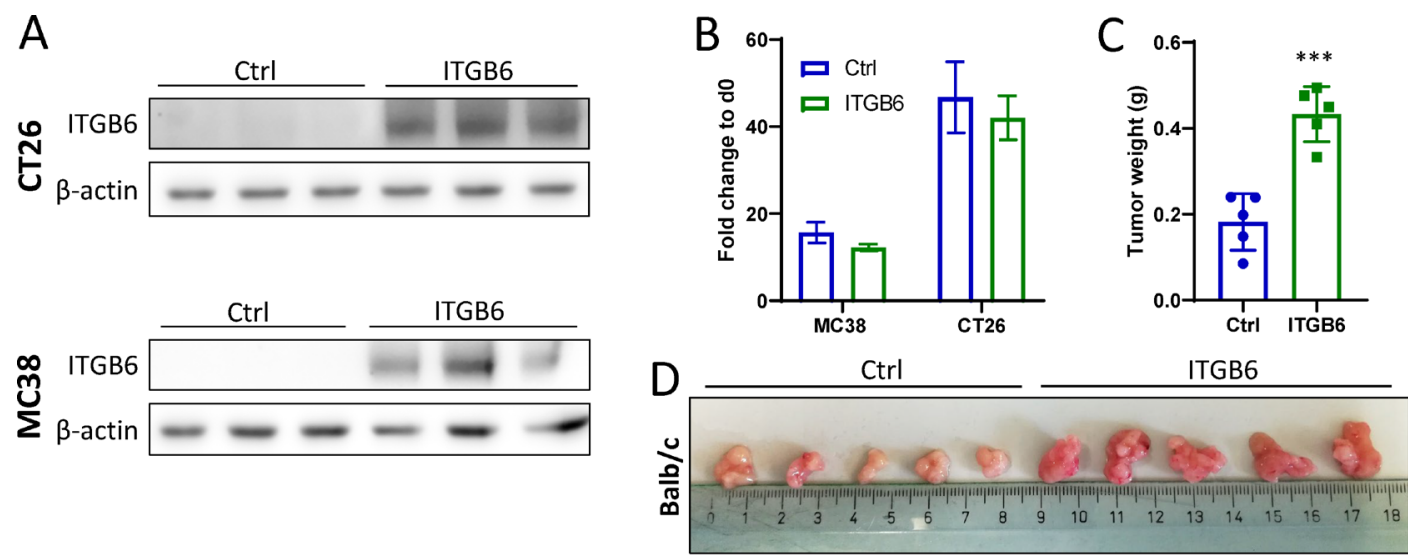

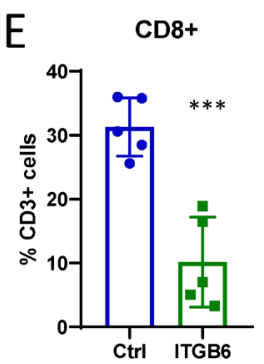

CD8+ TNF $\alpha+$

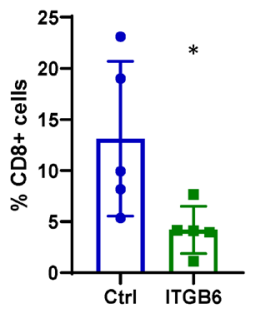

CD8+ IFN $\gamma+$

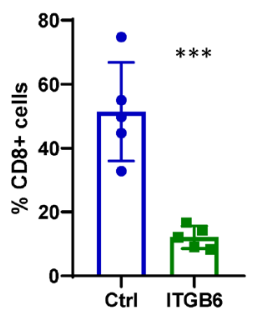

CD8+ GZMB+

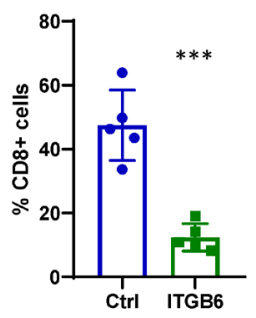

CD8+ PRF1+

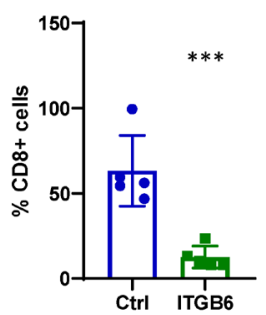

Ctrl ITGB6

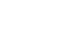

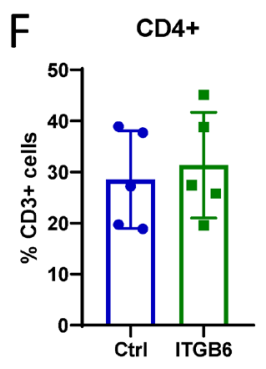

CD4+ TNF $\alpha+$

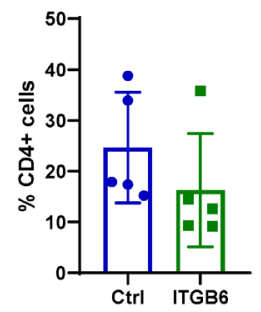

CD4+ IFN $\gamma+$

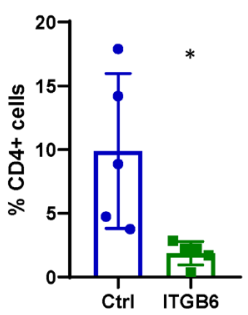

G Spleen CD4+ IFN $\gamma+$

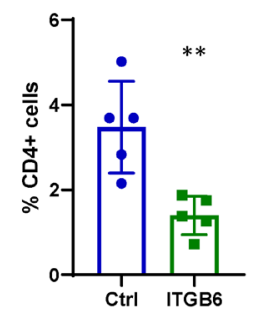

Spleen CD8+ IFN $\gamma+$

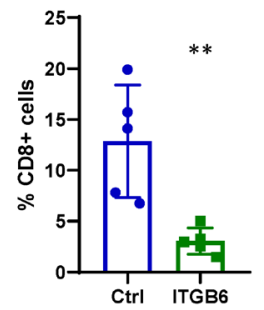

LN CD8+ IFNg+

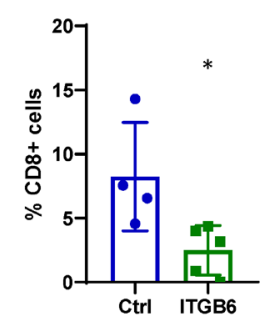

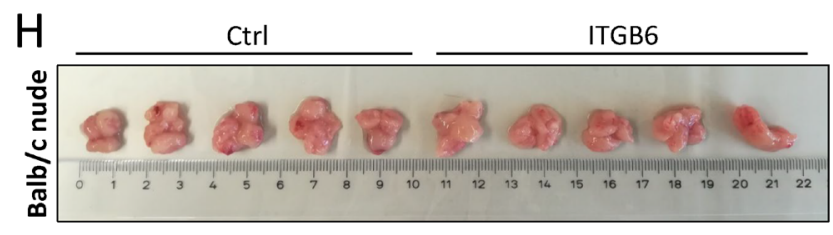

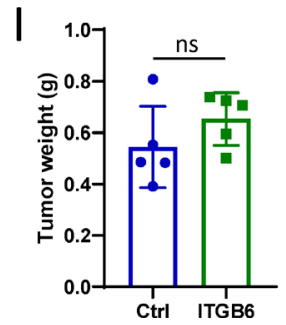

Figure 1 ITGB6 promotes tumor growth by inhibiting the T-cell antitumor immune response. (A) Western blot for ITGB6 in MC38 and CT26 cells that overexpress ITGB6. (B) 3-day proliferation assay with ITGB6 overexpressing and control cells. (C) CT26-ITGB6 and CT26-Ctrl cecum tumors at day 13 after injection into Balb/c mice. (D) Weight of CT26-ITGB6 and CT26-Ctrl cecum tumors at day 13 after injection. (E) Flow cytometry analysis of CD8+ Tcells isolated from tumor. (F) Flow cytometry analysis of CD4+ Tcells isolated from tumor. (G) Flow cytometry analysis of CD8+ and CD4+ Tcells isolated from spleen and lymph node (LN). (H) CT26-ITGB6 and CT26-Ctrl cecum tumors at day 13 after injection into Balb/c nude mice. (I) Weight of CT26-ITGB6 and CT26-Ctrl cecum tumors at day 13 after injection into Balb/c nude mice. Means and SDs are shown $(\mathrm{n}=5$ mice). Unpaired two-tailed t-test was used to calculate statistical significance. ns=not significant $(p \geq 0.05),{ }^{*} p<0.05,{ }^{* *} p<0.01$, ${ }^{\star \star \star} \mathrm{p}<0.001,{ }^{\star \star \star \star} \mathrm{p}<0.0001$. 
transduced with an empty control vector (Ctrl). Co-IP of ITGB6 and ITGAV showed that that the overexpressed ITGB6 is forming a heterodimer with endogenously expressed ITGAV (online supplemental figure S1B). Since ITGB6 expression has been reported to accelerate cell proliferation, ${ }^{33-35}$ we performed a 3-day proliferation assay in vitro to define the proliferation rate. However, neither CT26 nor MC38 cells showed upregulated proliferation on ITGB6 overexpression (figure 1B).

Next, to examine the effect of ITGB6 on intestinal tumor growth in vivo, we performed cecum injections of CT26-ITGB6 cells and CT26-Ctrl cells in Balb/c mice, which are syngeneic to the CT26 cell line. Mice injected with CT26-ITGB6 cells showed accelerated tumor growth, leading to larger and heavier tumors at the time of sacrifice (figure 1C,D). IHC stainings of the tumors for CD3, CD4, and CD8 showed significantly decreased T-cell numbers in ITGB6 tumors compared with Ctrl tumors whereas the proliferation marker Ki67 did not show any difference in tumor cell proliferation (online supplemental figure S1A). RT-qPCR analysis of the tumors revealed a massive upregulation of Itgb6 expression in CT26-ITGB6 tumors, causing decreased gene expression of the inflammatory cytokines tumor necrosis factor $\alpha($ Tnfa $)$ and interferon $\gamma($ Ifng), the cytolytic enzymes granzyme B $(G z m b)$ and perforin (Prf1), as well as the Th1 marker T-bet (Tbx21) (online supplemental figure S1C). Analysis of the T-cell compartment in the tumors by flow cytometry showed that the proportion of CD8+ T cells was reduced by ITGB6 (figure 1E). Similarly, granzyme B, perforin, IFN- $\gamma$ and TNF- $\alpha$ expression were strongly downregulated by ITGB6 in CD8+ T cells (figure 1E), whereas CD4+ T cells were not affected in their proportion, but showed reduced IFN- $\gamma$ expression (figure 1F). ITGB6 also downregulated the expression of IFN- $\gamma$ in CD8+ Tcells isolated from spleen and draining LNs and in splenic CD4+ T cells (figure 1G). For further analysis of the effect of ITGB6 expression in the tumor, we performed subcutaneous tumor injections. CT26-ITGB6 and CT26-Ctrl cells were injected into Balb/c mice and MC38-ITGB6 and MC38-Ctrl cells were injected into C57BL/6 mice, which are syngeneic to MC38 cells. Analogous to orthotopic injections, mice with CT26-ITGB6 or MC38-ITGB6 tumors showed a strongly enhanced tumor growth compared with mice with CT26Ctrl or MC38-Ctrl tumors (online supplemental figure S2A-C, E-G), as well as inhibition of the cytotoxic CD8+ Tcell response. Inhibition of CD8+ Tcell activation is indicated by decreased proportions of CD8+ cells within the CD3+ fraction and decreased expression of granzyme $\mathrm{B}$, perforin, IFN- $\gamma$ and TNF- $\alpha$ (online supplemental figure S2D,H). Similarly, CD4+ T cells showed decreased expression of IFN- $\gamma$ and TNF- $\alpha$ (online supplemental figure $\mathrm{S} 2 \mathrm{D}, \mathrm{H})$. Together with the inhibition of T-cell responses, gene expression of the chemokines CXCL9, CXCL10 and CXCL11, which are known to be involved in T-cell infiltration and activation as well as T-cell differentiation into the Th1 subtype, ${ }^{36}$ was suppressed in subcutaneous CT26-ITGB6 tumors (online supplemental figure S3A).
These data demonstrate that ITGB6 inhibits T-cells and promotes tumor growth independently of the tumor location or genetic background of the host.

To confirm that the tumor growth promoting effect of ITGB6 is mediated by T-cell inhibition, we injected CT26ITGB6 and CT26-Ctrl cells into Balb/c nude mice, which are lacking T-cells. In these mice, no significant difference in tumor weight was observed between CT26-ITGB6 and CT26-Ctrl tumors, indicating that tumor growth acceleration caused by ITGB6 is mediated by T-cell inhibition (figure 1H,I).

\section{Cytotoxic immune response regulation is the main function of ITGB6 within the tumor}

In order to obtain an overview over the functions of ITGB6 within the tumor, we performed RNA-seq transcriptomic analysis on subcutaneous MC38-ITGB6 and MC38-Ctrl tumors. Unsupervised hierarchical clustering of the observed DEGs grouped the biological replicates of MC38-ITGB6 and of MC38-Ctrl tumors into two distinct clusters (figure 2A). Many of the most significantly downregulated genes in MC38-ITGB6 tumors were T-cell markers (CD3d, CD3e, CD3g, CD8a,Trbc1, Trbc2) or genes involved in T-cell activation (Cxcl9, Txk, Itk) and cytotoxic functions (Gzmb, Gzma, Prfl) (figure 2B). We further investigated the function of the DEGs by GO enrichment analysis (figure 2C). The DEGs were mainly involved in biological processes related to the immune system and those were strongly downregulated in MC38ITGB6 tumors, indicating a strong effect of ITGB6 on the immune response. Therefore, besides cell adhesion, which also appears as one of the most affected biological processes, the main function of ITGB6 in the tumor appears to be the modulation of immune responses. Since adaptive as well as innate immune responses seem to be affected (figure 2C), we aimed to define changes in the proportions of different immune cell types in the tumors. An established computational method, using cell type-specific gene expression references, was used to deconvolute these cell type proportions from the bulk tissue RNA-seq data. While monocytes and regulatory T-cells (T-regs) were unaffected, proportions of several immune cell types, such as NK cells, B cells, M2 macrophages and neutrophils, were mildly affected by ITGB6 expression (online supplemental figure S3B). However, the proportion of CD8 T-cells within the tumors was strongly downregulated by ITGB6 (online supplemental figure S3B). Therefore, the main effect of ITGB6 in the antitumor immune response is the regulation of the cytotoxic T-cell reaction.

\section{T-cell inhibiting effect of ITGB6 is mediated through TGF- $\beta$ activation}

Phosphorylation of SMAD2 and SMAD3 was significantly upregulated in subcutaneous CT26-ITGB6 tumors compared with CT26-Ctrl tumors (figure 3A), demonstrating that integrin $\alpha v \beta 6$ activates TGF- $\beta$ signaling in the tumor. Likewise, analyzing our RNA-seq data for TGF- $\beta$ 
A

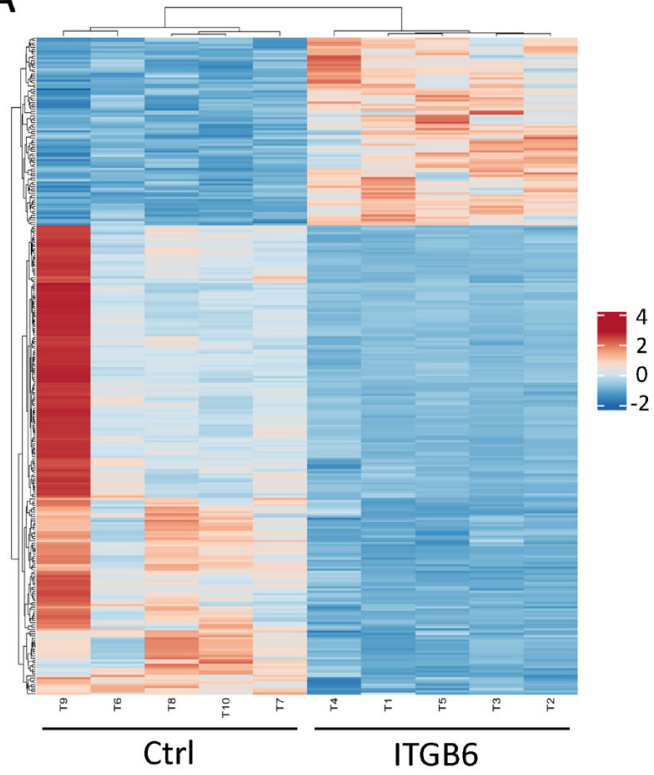

C

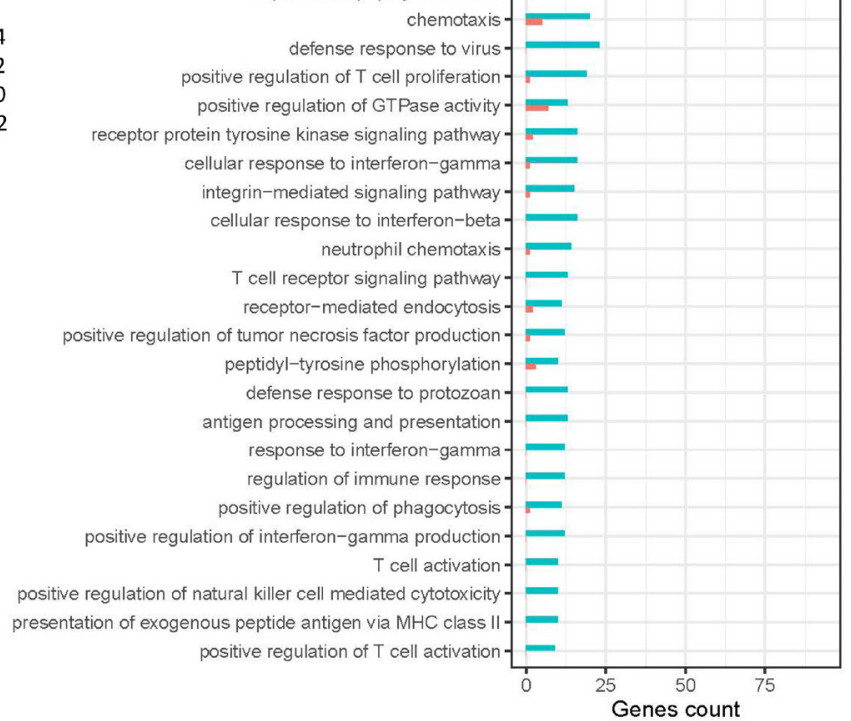

B

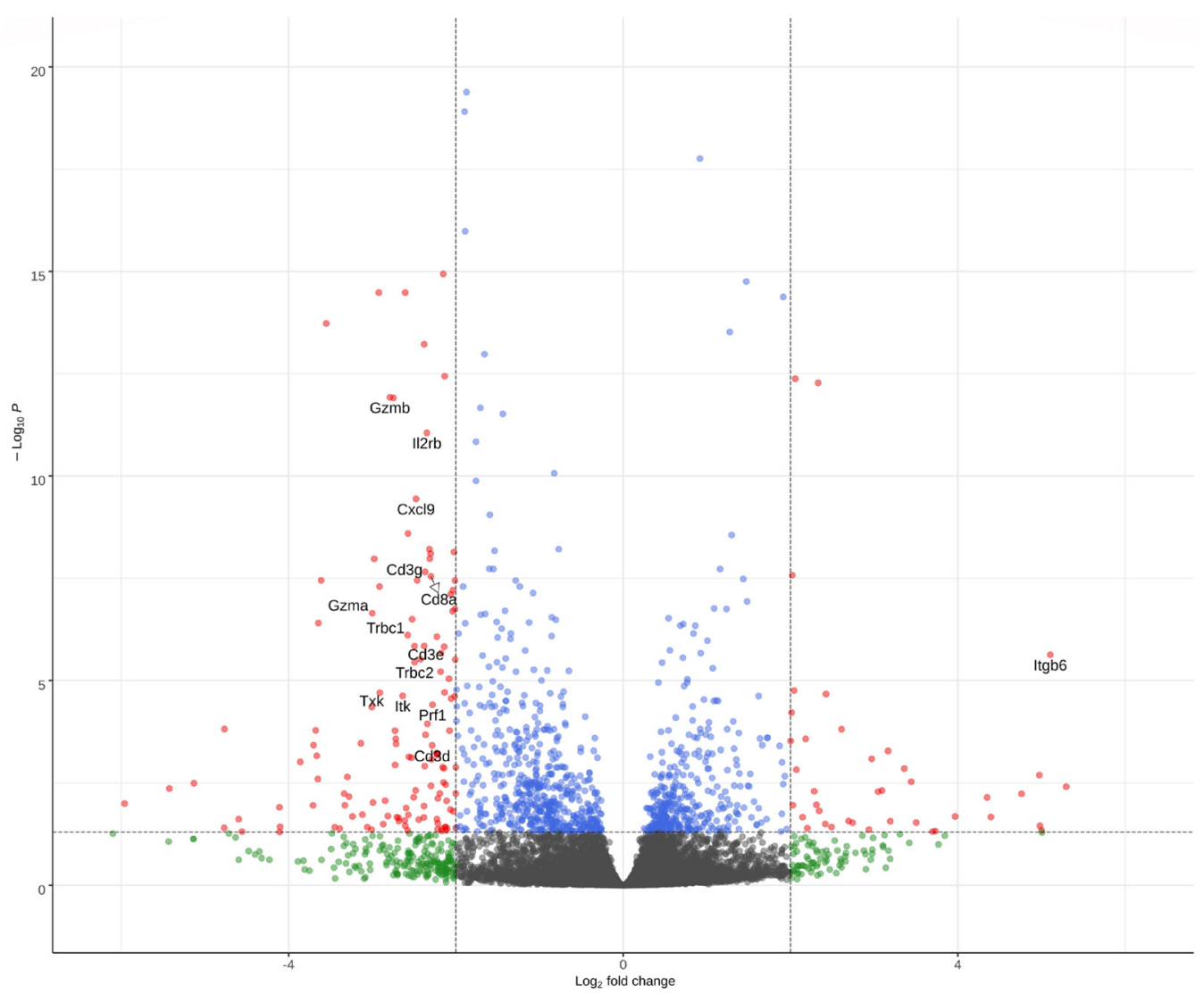

Figure 2 Cytotoxic immune response regulation is the main function of ITGB6 within the tumor. (A) Heatmap of differentially expressed genes (DEGs) $(p<0.001)$ in MC38-ITGB6 and MC38-Ctrl tumors, generated by unsupervised hierarchical clustering ( $n=5$ mice). (B) Volcano plot displaying DEGs from MC38-ITGB6 versus MC38-Ctrl tumors. Y-axis corresponds to $p$ value of $-\log 10$. X-axis displays log 2 -fold change value. Indicated limits represent $D E G s$ with $p<0.05$ and $\log 2$ fold change above +2 or below -2. (C) Gene ontology enrichment analysis of DEGs. Bars in red or blue indicate the number of genes involved in upregulation or downregulation of the respective biological process in ITGB6-expressing tumors. 
A

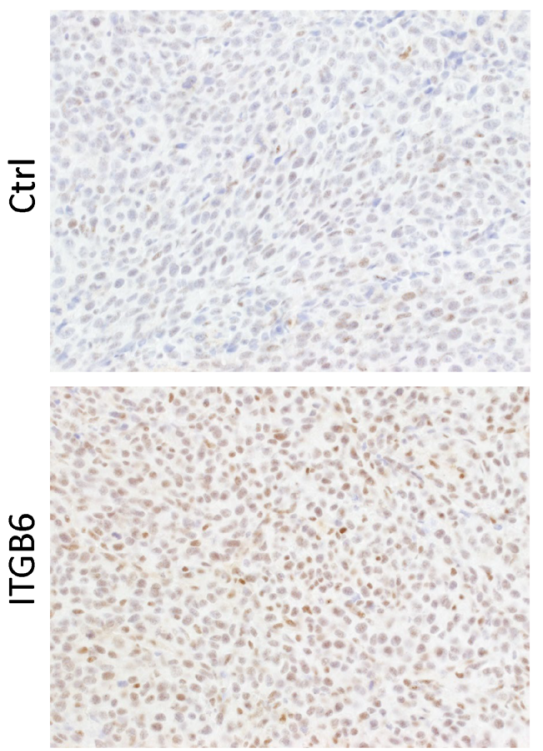

pSmad2
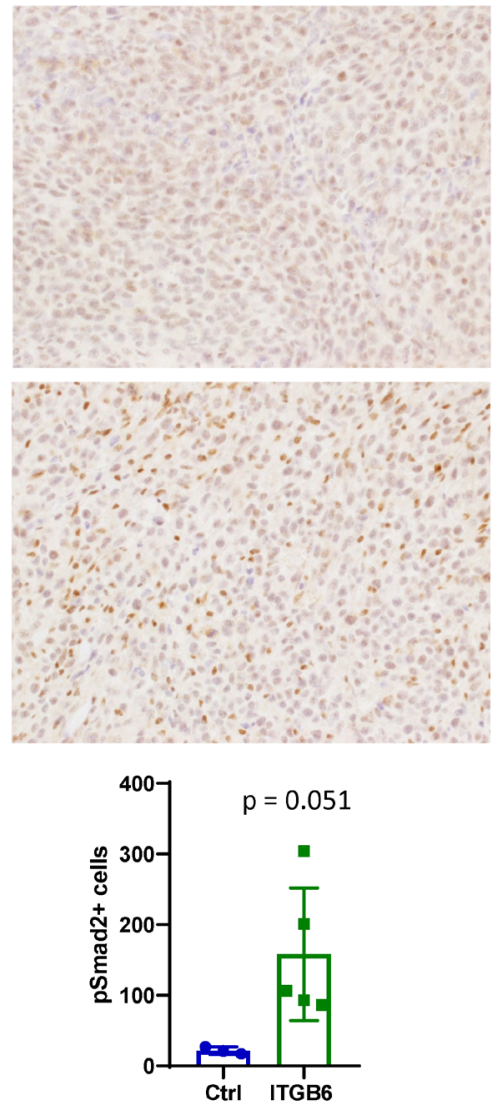

SOX4
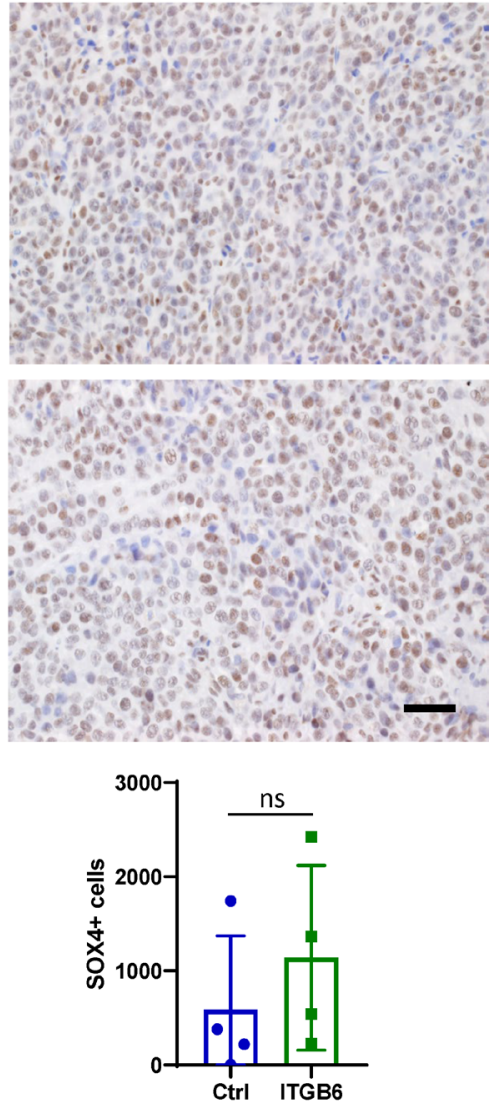

B

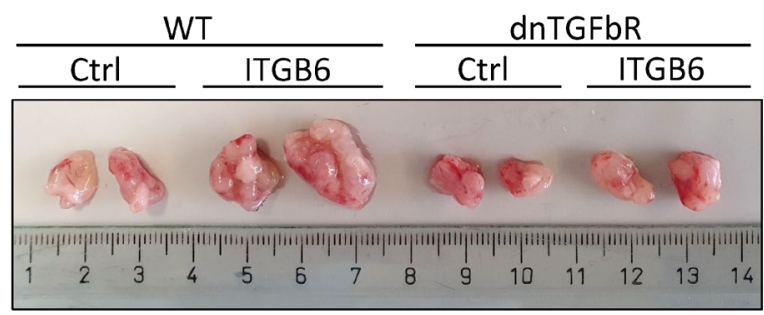

C

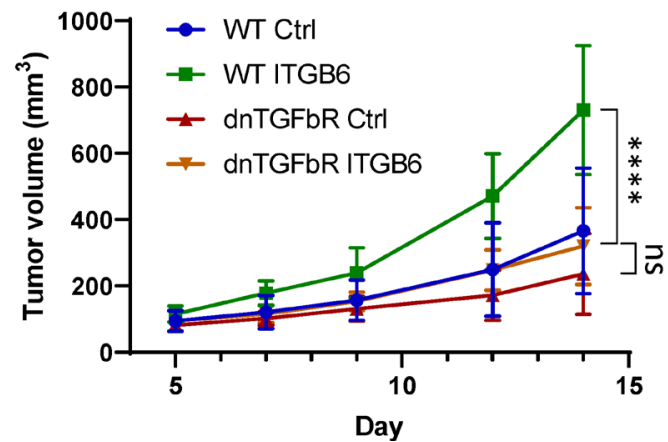

Figure 3 T-cell inhibiting effect of ITGB6 is mediated through TGF- $\beta$ activation. (A) Immunohistochemical (IHC) stainings for pSmad2, pSmad3 and SOX4 in subcutaneous CT26-ITGB6 and CT26-Ctrl tumors. Representative images of IHC stainings (top) and quantification of the number of stained cells (below). Scale bar=50 $\mu \mathrm{m}$. (B) Representative image of subcutaneous CT26ITGB6 and CT26-Ctrl tumors grown in CD4-dnTGFBR2 mice or C57BL/6 WT mice at day 14 after injection. (C) Tumor volume development of CT26-ITGB6 and CT26-Ctrl tumors grown in CD4-dnTGFBR2 mice or C57BL/6 WT mice. (D) Weight of CT26ITGB6 and CT26-Ctrl tumors grown in CD4-dnTGFBR2 mice or C57BL/6 WT mice at day 14 after injection. Means and SDs are shown. Unpaired two-tailed t-test (A) ( $n=5$ mice), one-way analysis of variance (ANOVA). (D) and two-way ANOVA (C) with Tukey's post-hoc test ( $n=5$ mice, 2 tumors per mouse) were used to calculate statistical significance. $n s=n o t$ significant $(p \geq 0.05)$, ${ }^{*} \mathrm{p}<0.05,{ }^{* *} \mathrm{p}<0.01,{ }^{* * *} \mathrm{p}<0.001,{ }^{* * *} \mathrm{p}<0.0001$. 
response signatures in fibroblasts (F-TBRS) and T-cells $(\mathrm{T}-\mathrm{TBRS})^{37} 38$ demonstrated increased levels of TGF- $\beta$ signaling in MC38-ITGB6 tumors compared with MC38Ctrl tumors (online supplemental figure S3C). We also observed an upregulation of CALD1 and IGFBP7, two TGF- $\beta$ induced factors expressed in stomal cells of the tumor, which predict poor prognosis ${ }^{38}$ (online supplemental figure S3D). However, we did not observe a significant upregulation of SOX4 (figure 3A), a TGF- $\beta$ target gene that has been reported to inhibit the T-cell response when expressed by the tumor cells in TNBC. ${ }^{21}$ To further investigate the role of $\alpha \mathrm{v} \beta 6$-mediated TGF- $\beta$ activation in the T-cell tumor response, we subcutaneously injected CT26-ITGB6 and CT26-Ctrl cells into CD4-dnTGFBR2 transgenic mice, which express a dominant-negative form of TGFBR2 selectively in CD4+ and CD8+T cells. These transgenic mice exhibit blocked TGF- $\beta$ signaling specifically and exclusively in CD4+ and CD8+ T cells. ${ }^{39}$ In CD4-dnTGFBR2 mice, no difference in tumor weight or volume was observed between CT26-ITGB6 and CT26-Ctrl tumors, whereas in wildtype (WT) mice CT26ITGB6 tumors showed significantly increased growth (figure 3B-D). Furthermore, CT26-ITGB6 tumors grew significantly larger in WT mice compared with the same tumors in CD4-dnTGFBR2 mice (figure 3B-D). Together, these results show that the tumor growth promoting and T-cell inhibiting effect of ITGB6 is mediated by increased TGF- $\beta$ signaling in T-cells.

\section{ITGB6 expression leads to local, but not systemic T-cell inhibition}

T-cells isolated from spleens and LNs of mice bearing ITGB6 expressing tumors showed significantly decreased IFN- $\gamma$ expression compared with the ones from mice with control tumors (figure 1G), suggesting that ITGB6 expression in the tumor might cause systemic T-cell inhibition. Therefore, we examined if T-cell inhibition provoked by ITGB6-tumors affects the growth of Ctrl tumors within the same host. For that purpose, we subcutaneously injected CT26-ITGB6 tumors in one flank and CT26-Ctrl tumors in the other flank of the same mouse and compared tumor growth and T-cell activity in these tumors to their respective counterparts in mice only bearing CT26-ITGB6 or CT26-Ctrl tumors (figure 4A). Tumor size of CT26-Ctrl tumors (Mix Ctrl) was not affected by the presence of CT26-ITGB6 tumors (figure 4B,C). Similarly, the CT26ITGB6 tumors did not affect the CD8+ T cell proportion, cytotoxic activity, Th1 differentiation or T-cell infiltration in the CT26-Ctrl tumor (Mix Ctrl) in the same mouse (figure 4D, online supplemental figure S4). Likewise, the presence of CT26-Ctrl tumors and concomitant T-cell activation did not inhibit the growth or enhance T-cell infiltration or T-cell activity in CT26-ITGB6 tumors (Mix ITGB6) (figure 4B, online supplemental figure S4). Immunofluorescent staining from the boundary of CT26ITGB6 tumors revealed that CD8+ Tcells were mainly present outside the tumor mass where only little pSmad3 is detected and were not able to infiltrate into the tumor tissue with more pSmad3 expression (figure 4E). These T-cells surrounding the tumor mass showed undetectable or very low pSmad3 staining (figure 4E). Very few CD8 T-cells were present in the center of these tumors and showed higher pSmad3 expression (figure 4E). These data indicate that CD8 T-cells are locally inactivated and blocked from infiltrating the tumor mass by direct TGF- $\beta$ signaling in T-cells. Thus, systemically activated T-cells are ineffective against ITGB6-expressing tumors and T-cell inhibition through ITGB6 expression is acting only locally in ITGB6-expressing tumors and is not transmitted to tumors with low levels of ITGB6 expression. This creates a direct selective pressure that selects towards tumor cells with elevated expression of ITGB6, enabling them to escape the cytotoxic T-cell response.

\section{Integrin $\alpha \mathrm{V} \beta 6$ blockade sparks T-cell antitumor response and overcomes resistance to CBT}

After demonstrating that expression of integrin $\alpha v \beta 6$ is an immune evasion strategy of tumor cells, we examined if integrin $\alpha v \beta 6$ can be effectively targeted to induce T-cell antitumor responses. For that purpose, we subcutaneously injected CT26-ITGB6 tumors and treated the mice with either the $\alpha v \beta 6$ neutralizing antibody 6.8G6 ( $\alpha$ ITGB6) or isotype control antibody (IgG) (figure 5A). Tumor size was significantly decreased after treating the tumors with $\alpha$ ITGB6 for 12 days (figure 5B-D). Moreover, inhibition of $\alpha v \beta 6$ significantly increased the proportion of CD8+ Tcells and the expression of the cytolytic enzymes granzyme $\mathrm{B}$ and perforin (figure $5 \mathrm{E}$ ). Interestingly, $\alpha$ ITGB6 treatment also led to heavily increased expression of the immune checkpoint molecules CTLA-4 and PD-1 (figure 5E), suggesting that T-cell antitumor response might be further enhanced with CBT. Therefore, we combined $\alpha$ ITGB6 treatment with a PD-1 blocking antibody ( $\alpha$ PD-1) in a subsequent experiment (figure 5F). Notably, ITGB6 expressing tumors seemed to be resistant to CBT since treatment with $\alpha \mathrm{PD}-1$ alone did not have any effect on tumor growth (figure 5G-I). However, while $\alpha$ ITGB6 treatment alone decreased tumor growth significantly, the combination with $\alpha \mathrm{PD}-1$ caused an even more potent immune response leading to tumor shrinkage (figure 5G-I). While there was no effect on the T-cell response by $\alpha \mathrm{PD}-1$ treatment alone (figure $5 \mathrm{~J}$ ), combination treatment triggered similar granzyme $\mathrm{B}$, perforin and T-bet expression levels as $\alpha$ ITGB6 alone and the proportion of CD8+ T cells in the tumor was even further increased (figure 5J). CD4+ Tcell proportion in the tumor was not affected by $\alpha$ ITGB 6 or the combination treatment (figure 5J). Splenic CD8+ and CD4+ T cells showed elevated expression of IFN- $\gamma$ and TNF- $\alpha$ as well as T-bet on $\alpha$ ITGB6 treatment, which was further enhanced in mice treated with $\alpha$ ITGB 6 and $\alpha$ PD-1 (online supplemental figure S5A). Both treatment modalities also led to higher expression of the activation marker CD44 in splenic T-cells (online supplemental figure S5A). IHC staining of pSmad3, CD3, CD4 and CD8 revealed decreased TGF- $\beta$ signaling and a higher abundance of 
A

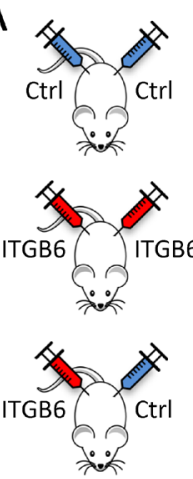

D

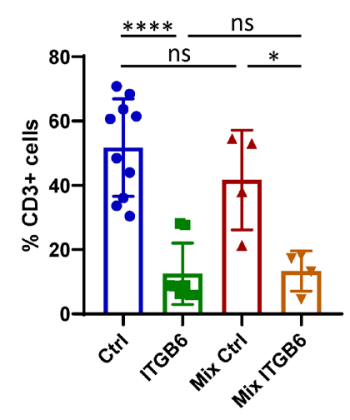

CD8+ GZMB+ Perforin+
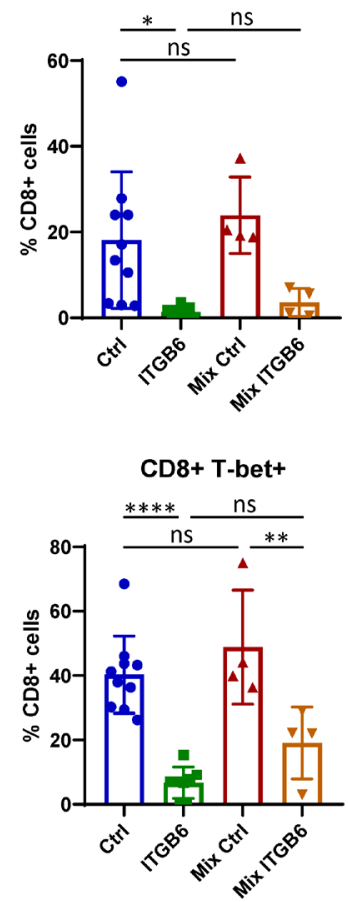

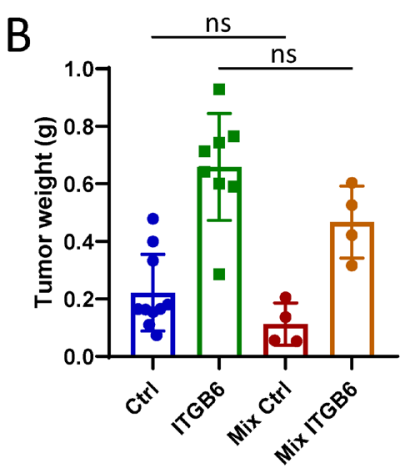

C

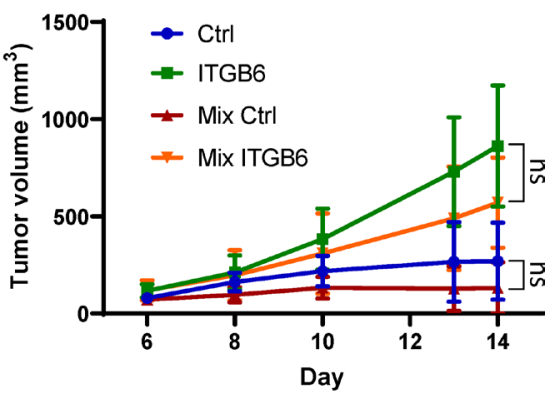

$\mathrm{E}$

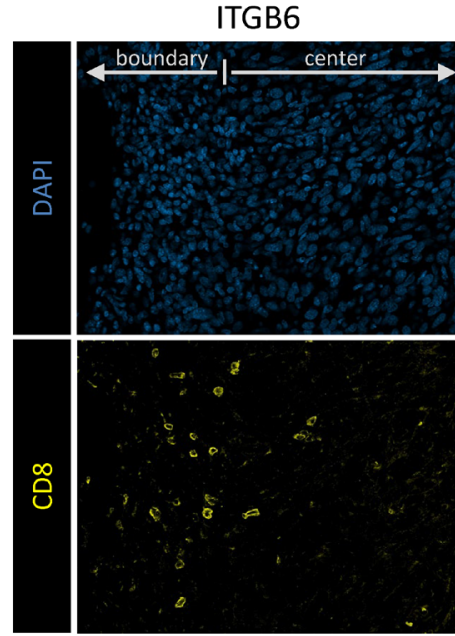

Mix ITGB6
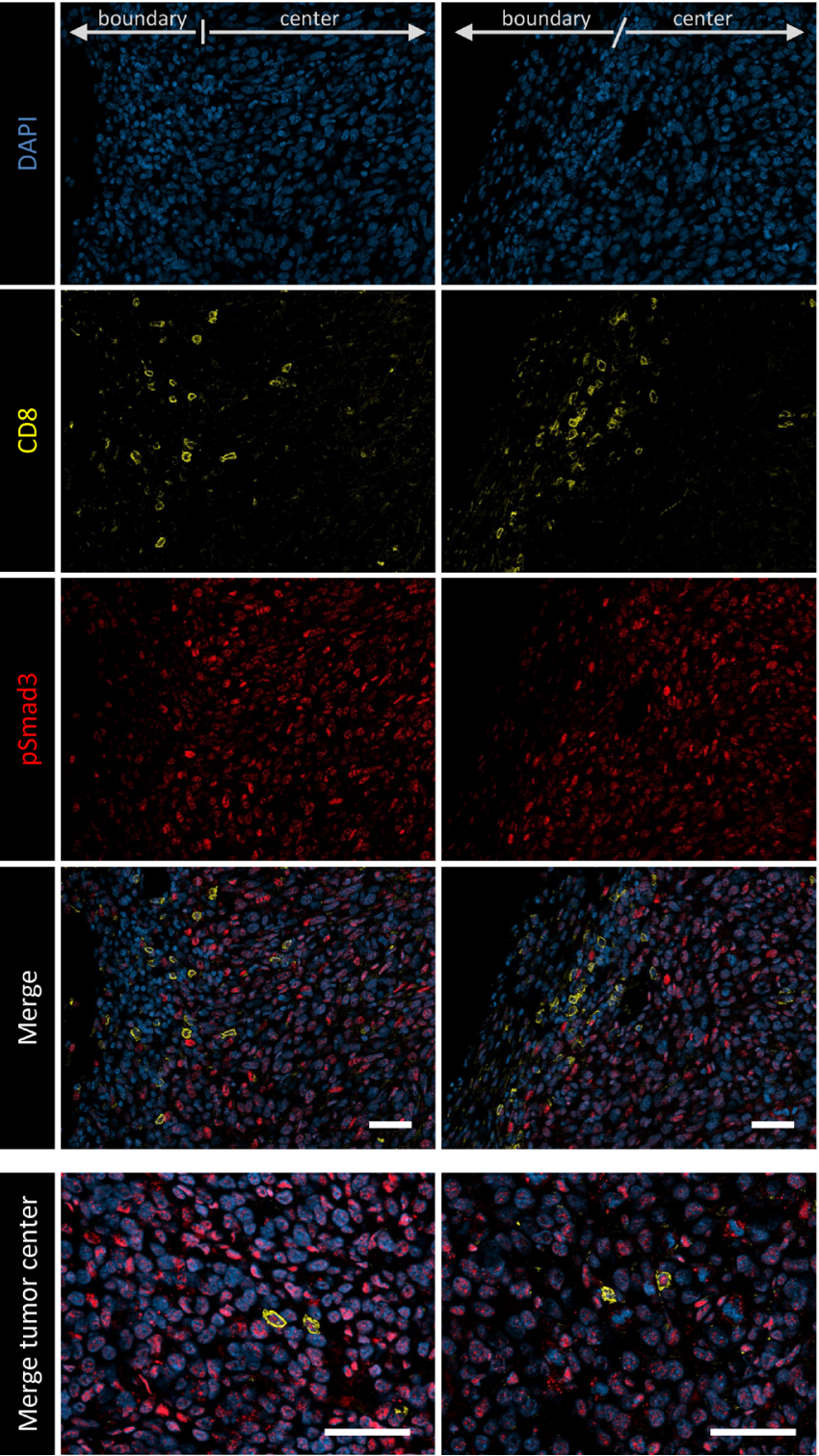

Figure 4 ITGB6 expression leads to local, but not systemic T-cell inhibition. (A) Experimental design of injection scheme. Subcutaneous injection of CT26-Ctrl tumors or CT26-ITGB6 tumors in both flanks or CT26-Ctrl tumors in one flank and CT26ITGB6 tumors in the other flank of the mice (Mix). (B) Weight of tumors from mice bearing only CT26-ITGB6 or CT26-Ctrl tumors or mice bearing both tumors (Mix). (C) Tumor volume development of tumors from mice bearing only CT26-ITGB6 or CT26-Ctrl tumors or mice bearing both tumors (Mix). (D) Flow cytometry analysis of T-cells isolated from tumors of mice bearing only CT26-ITGB6 or CT26-Ctrl tumors or mice bearing both tumors (Mix). (E) Immunofluorescent stainings for CD8 and pSmad3 in CT26-ITGB6 tumors from mice bearing only CT26-ITGB6 tumors or mice bearing both tumors (Mix). Means and SDs are shown $(n=5$ mice). One-way analysis of variance (ANOVA) (B and D) and two-way ANOVA (C) with Tukey's post-hoc test were used to calculate statistical significance. ns $=$ not significant $(p \geq 0.05),{ }^{*} p<0.05,{ }^{* \star} p<0.01,{ }^{* \star *} p<0.001,{ }^{* \star * *} p<0.0001$. 
A Tumor cell

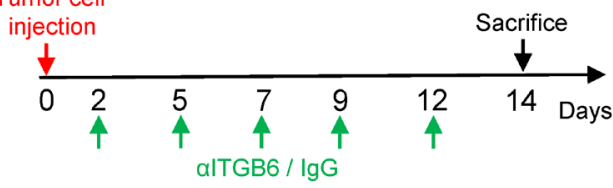

B
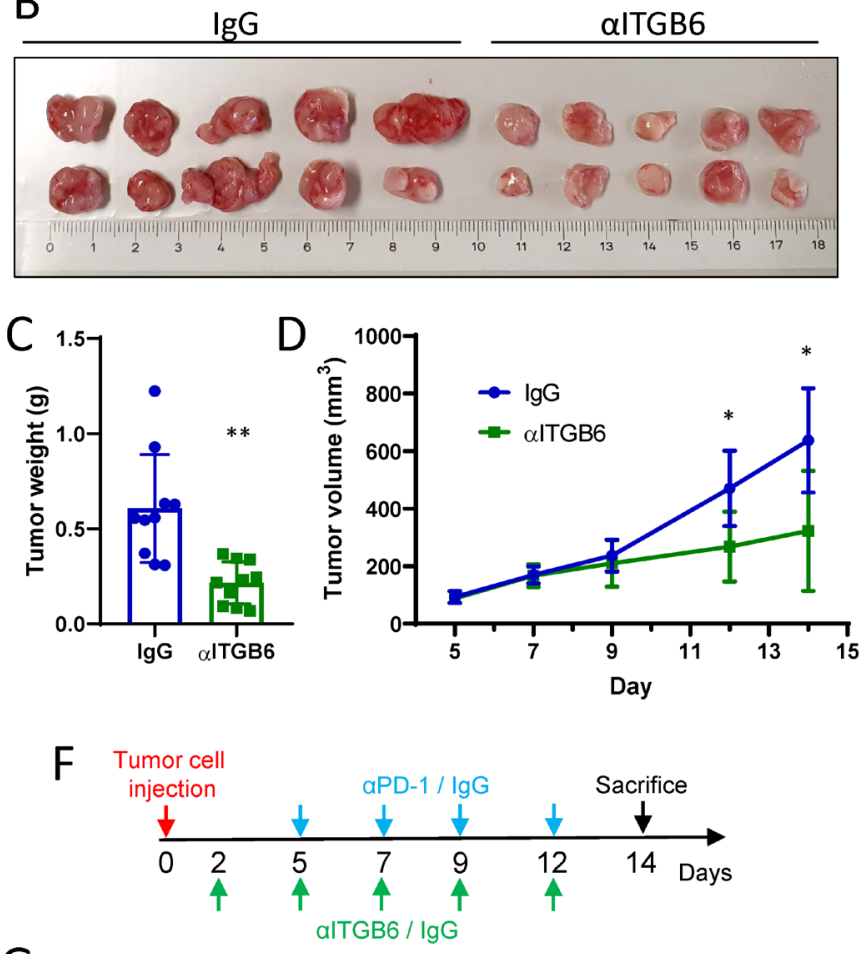

G
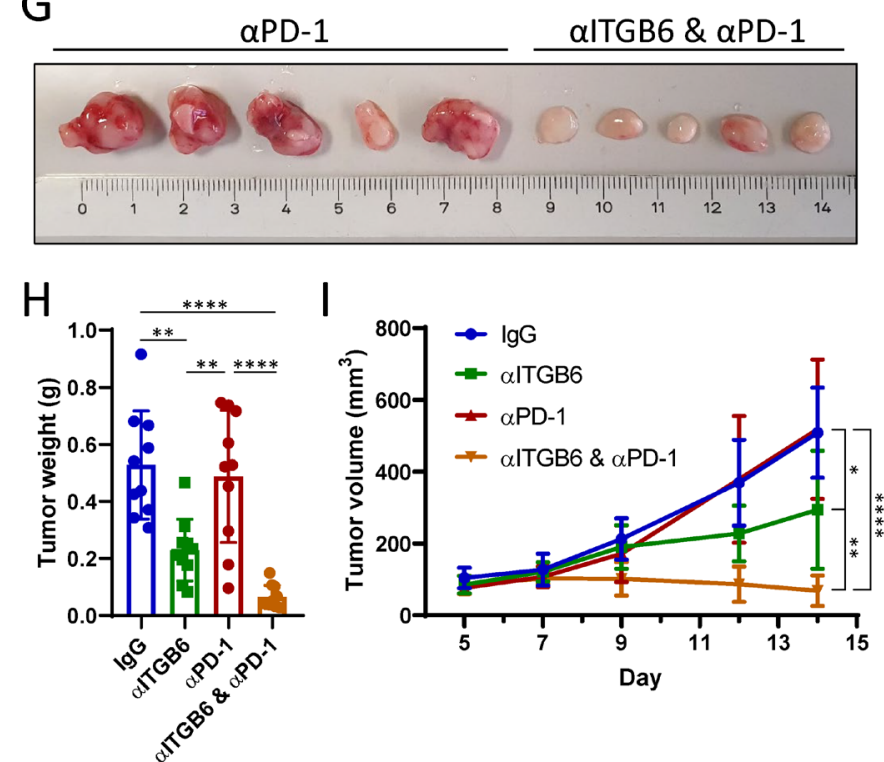
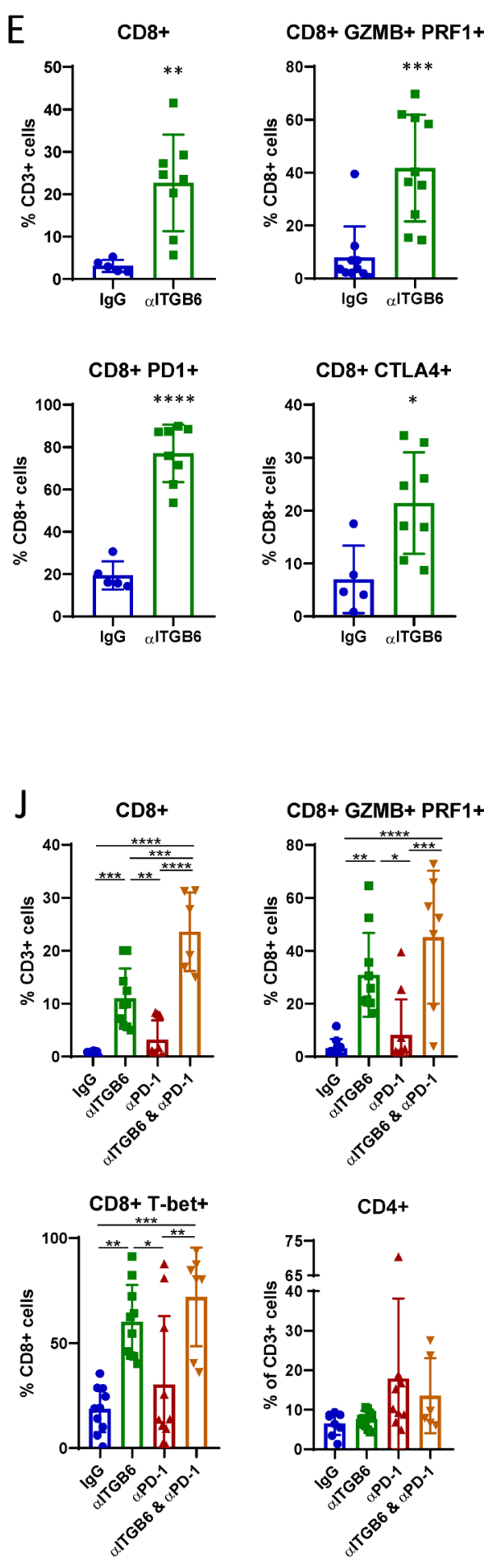

Figure 5 Integrin $\alpha v \beta 6$ blockade sparks T-cell antitumor response and overcomes resistance to CBT. (A) Experimental design of $\alpha$ ITGB6 antibody (6.8G6) administration. (B) Subcutaneous CT26-ITGB6 tumors treated with $\alpha$ ITGB6 or IgG control. (C) Tumor weight of subcutaneous CT26-ITGB6 tumors treated with alTGB6 or IgG control. (D) Tumor volume development of subcutaneous CT26-ITGB6 tumors treated with $\alpha$ ITGB6 or IgG control. (E) Flow cytometry analysis of CD8+ Tcells in CT26-ITGB6 tumors treated with $\alpha$ ITGB6 or IgG control. (F) Experimental design of $\alpha$ ITGB6 (6.8G6) and $\alpha$ PD-1 antibody administration. (G) Representative image of subcutaneous CT26-ITGB6 tumors treated with $\alpha$ PD-1 or $\alpha$ ITGB6 and $\alpha$ PD-1. (H) Tumor weight of subcutaneous CT26-ITGB6 tumors treated with $\alpha$ ITGB6, $\alpha$ PD-1, $\alpha$ ITGB6 and $\alpha$ PD-1 or IgG control. (I) Tumor volume development of subcutaneous CT26-ITGB6 tumors treated with $\alpha$ ITGB6, $\alpha$ PD-1, $\alpha$ ITGB6 and $\alpha \mathrm{PD}-1$ or IgG control. (J) Flow cytometry analysis of CD8+ T cells in CT26-ITGB6 tumors treated with $\alpha$ ITGB6, $\alpha$ PD-1, $\alpha$ ITGB6 and $\alpha$ PD-1 or lgG control antibody. Means and SDs are shown ( $n=5$ mice, 2 tumors per mouse). Unpaired two-tailed t-test (C, E) one-way analysis of variance (ANOVA) $(\mathrm{H}, \mathrm{J})$ and two way ANOVA ( $\mathrm{D}$ and $\mathrm{I})$ with Tukey's post-hoc test were used to calculate statistical significance. ${ }^{*} \mathrm{P}<0.05,{ }^{* \star} \mathrm{p}<0.01,{ }^{\star \star \star} \mathrm{p}<0.001,{ }^{* \star \star \star} \mathrm{p}<0.0001$. 
CD8+ Tcells in $\alpha$ ITGB6-treated tumors (online supplemental figure S5B, S6). Together, these data show that ITGB6 expression causes resistance to $\alpha \mathrm{PD}-1$ therapy, which can be overcome by combining CBT with $\alpha v \beta 6$ blockade to provoke a potent T-cell antitumor response.

In a next step, we examined if $\alpha \mathrm{PD}-1$ therapy efficacy can also be improved with a different $\alpha v \beta 6$-blocking antibody (6.3G9) to exclude that the antitumor effect is specific to the $\alpha$ ITGB 6 antibody that we previously used (6.8G6) or specific to the epitope targeted by that antibody. ${ }^{24}$ Differences in tumor size as well as cytotoxic CD8+ Tcell activity showed the same additive effects of 6.3G9 together with $\alpha \mathrm{PD}-1$ therapy as observed before with 6.8G6 (online supplemental figure S7A-E). Additionally, staining for Ki67 in this experiment showed that $\alpha$ ITGB6 and $\alpha$ PD- 1 combination treatment strongly enhances the proliferation of CD8+ Tcells in the tumors (online supplemental figure $\mathrm{S} 7 \mathrm{E}$ ), which might partly explain the observed increase of CD8+ T cell numbers. In conclusion, $\alpha \mathrm{PD}-1$ therapy efficacy might be enhanced with any integrin $\alpha v \beta 6$-inhibiting agent and is not antibody-dependent or epitope-dependent.

We further investigated whether the efficacy of the chemotherapeutic drug 5-FU, one of the most commonly used agents in CRC treatment, ${ }^{40}$ can be enhanced by aITGB6 (6.8G6) administration. For that purpose, we compared the effect of 5-FU or $\alpha$ ITGB6 administration alone to the combination of 5-FU and $\alpha$ ITGB6 in subcutaneous CT26-ITGB6 tumors (online supplemental figure S8A). All treatment modalities inhibited tumor growth with no significant differences between the three treatment groups (online supplemental figure S8A-D). Thus, in CT26-ITGB6 tumors, $\alpha$ ITGB6 is as efficient in controlling tumor growth as 5-FU, whereas the combination of them does not provoke an additive effect. The inability of $\alpha$ ITGB6 to enhance 5-FU treatment efficiency might be explained by the T-cell inhibiting effect of 5-FU administration. While CD8+ T cells are abundantly present and produce high amounts of granzyme B and perforin in the $\alpha$ ITGB6 treated group, this T-cell activating effect was eradicated on combination with 5-FU (online supplemental figure S8E). These data are in accordance with previous studies, which show that repeated 5-FU application impairs T-cell antitumor immune functions. ${ }^{41}$ Therefore, efficacy of $\alpha$ ITGB 6 treatment, or any other T-cell activating therapy, might be impaired by combined administration with the chemotherapeutic drug 5-FU.

\section{Integrin $\alpha v \beta 6$ blockade overcomes resistance to $\mathrm{CBT}$ in tumors without artificial ITGB6 overexpression}

So far, our data have demonstrated that combination treatment with $\alpha$ ITGB 6 and $\alpha$ PD- 1 is effective in ITGB6 overexpressing tumors with artificially enhanced ITG6 expression levels. Whereas this model is useful to study the effects of ITGB 6 on tumor growth and immune response, its vigorous ITGB6 expression cannot be compared with ITGB6 levels in tumors that naturally develop from somatic cells and upregulate ITGB6 expression through a selective process. To find a model that resembles ITGB6 expression of a human tumor more closely, we assessed endogenous Itgb6 expression in the murine mammary carcinoma cell line 4T1. The 4T1 cell line exhibited high endogenous Itgb6 expression when compared with CT26 or MC38 cells, yet Itgb6 expression in CT26-ITGB6 and MC38-ITGB6 cells was 150-300 times higher than in 4T1 cells (figure 6A). Similarly, when injected as subcutaneous tumors, 4T1 tumors expressed substantially less Itgb6 than CT26-ITGB6 and MC38-ITGB6 tumors (figure 6B). We therefore examined the efficacy of the $\alpha$ ITGB6 (6.8G6) and $\alpha$ PD-1 combination treatment in subcutaneously injected 4T1 tumors (figure 6C). As seen before in ITGB6 overexpressing tumors, $\alpha$ PD- 1 treatment alone did not have any effect on tumor growth, indicating that even a low level of ITGB6 expression is enough to confer CBT resistance (figure $6 \mathrm{D}, \mathrm{E}$ ). However, $\alpha v \beta 6$ blockade overcame this resistance in $4 \mathrm{~T} 1$ tumors and boosted $\alpha \mathrm{PD}-1$ therapy efficacy, leading to significantly reduced tumor size compared with isotype control IgG-treated tumors (figure 6D,E). Therefore, $\alpha$ ITGB6 and $\alpha$ PD-1 combination therapy is effective to treat tumors with moderate ITGB6 expression levels.

\section{Integrin $\alpha \mathrm{V} \beta 6$ inhibits T-cell immune response in the majority of patients with CRC}

Having shown that ITGB6 inhibits the T-cell antitumor response and accelerates tumor growth in mice, we investigated the effect of ITGB6 expression in human tumors. For that purpose, we analyzed ITGB6 expression in tumor specimen of a prospectively collected cohort of 343 patients with colon carcinoma by RT-qPCR. Disease free survival was significantly decreased in patients with high ITGB6 expression in the tumor, indicating that ITGB6 promotes a more aggressive disease course (figure 7A). IHC stainings of human CRC tumors with high or low ITGB6 expression revealed that there is no association of SOX 4 staining intensity and ITGB6 expression, as it was shown in TNBC cells $^{21}$ (online supplemental figure S8F). To examine whether ITGB6 is affecting the T-cell response in human CRC tumors, we compared ITGB6 and T-cell response marker expression in RNAseq data from the TCGA-COAD dataset, which contains data from 521 patients with CRC. ITGB6 expression levels were widely varying between patients (figure 7B). However, by ordering the patients according to their ITGB6 expression, we found a particularly pronounced decrease of ITGB6 levels in the 50 patients with lowest ITGB6 expression compared with the remaining patients (figure 7B). Therefore, we compared T-cell response marker expression of the 50 patients with lowest ITGB6 expression (low) to the remaining 471 patients (high) (figure 7C). Similar to the observation in the RNAseq from the mouse tumors, the T-cell markers $C D 8 a, C D 3 d, C D 3 e, T R B C 2, I l 2 R b$ as well as the cytotoxicity markers GZMA, GZMB, and PRF1 were significantly upregulated in the group with low ITGB6 expression (figure 7C). Likewise, expression of the marker for Th1 T-cell differentiation $T B X 21$, the cytokine $I F N \gamma$, as well as the chemokine 
A

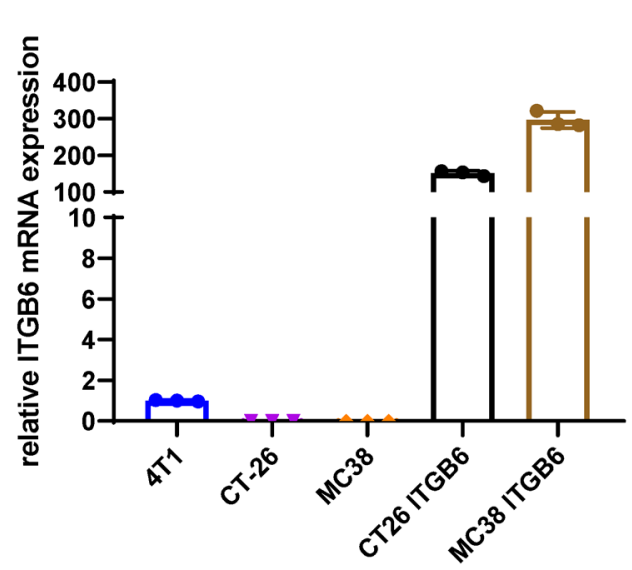

B

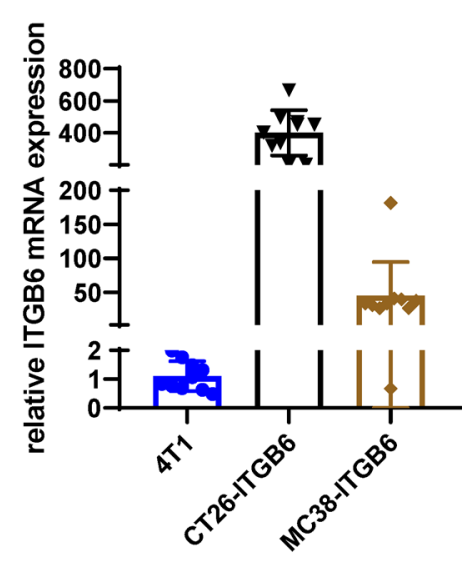

C

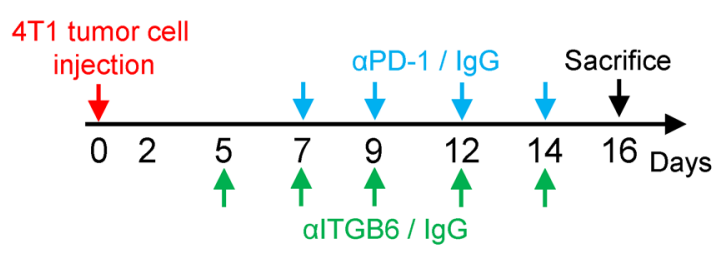

D

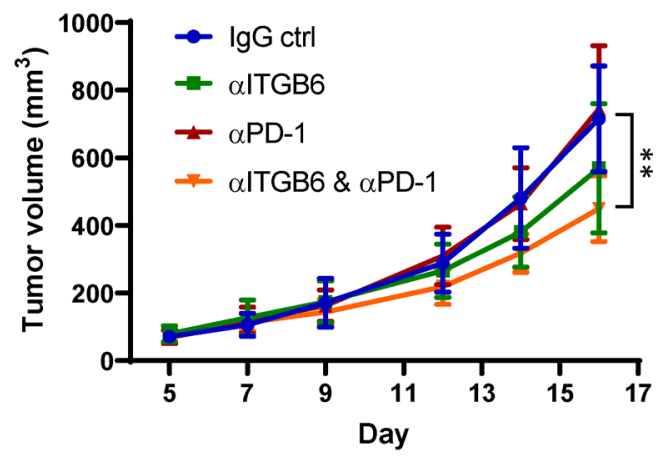

$\mathrm{E}$

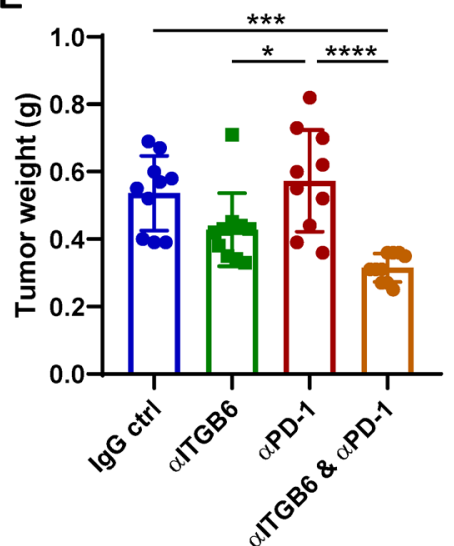

Figure 6 Integrin $\alpha v \beta 6$ blockade overcomes resistance to checkpoint blockade therapy in tumors without artificial ITGB6 overexpression. (A) RT-qPCR analysis of Itgb6 expression in murine carcinoma cell lines. (B) RT-qPCR analysis of Itgb6 expression in tumors grown from 4T1, CT26-ITGB6 and MC38-ITGB6 cells. (C) Experimental design of $\alpha$ ITGB6 (6.8G6) and $\alpha \mathrm{PD}-1$ antibody administration. (D) Tumor volume development of subcutaneous 4T1 tumors treated with $\alpha \mathrm{ITGB6}, \alpha \mathrm{PD}-1$, $\alpha$ ITGB6 and $\alpha$ PD-1 or IgG control. (E) Tumor weight of subcutaneous 4T1 tumors treated with $\alpha$ ITGB6, $\alpha$ PD-1, $\alpha$ ITGB6 and $\alpha P D-1$ or IgG control. Means and SDs are shown ( $n=5$ mice, 2 tumors per mouse). One-way analysis of variance (ANOVA) (E) and two way ANOVA (D) with Tukey's post-hoc test were used to calculate statistical significance. ${ }^{*} p<0.05,{ }^{* *} p<0.01$, ${ }^{* \star *} p<0.001,{ }^{* * * *} p<0.0001$.

CXCL9 were higher in that group (figure 7C). Remarkably, the patients with high ITGB6 expression showed decreased PD-1 expression, suggesting that ITGB6 confers resistance to $\alpha \mathrm{PD}-1$ treatment in human patients (figure $7 \mathrm{C}$ ). Since only the samples with lowest ITGB6 expression show an increase in T-cell response marker expression, most patients appear to have sufficient ITGB6 expression to provoke T-cell inhibition. Therefore, the vast majority of patients would qualify for $\alpha$ ITGB 6 treatment to overcome immune response evasion mediated by ITGB6.

\section{DISCUSSION}

Our data demonstrate that upregulation of $\alpha v \beta 6$ expression represents an effective immune evasion strategy by which tumor cells are able to escape the T-cell anticancer immune response. We showed that tumorous integrin $\alpha v \beta 6$ activates TGF- $\beta$ signaling in T-cells, thereby reducing cytotoxic CD8+ T cell activity and infiltration. Our results very closely resemble the TGF- $\beta$-mediated inhibition of T-cells shown in previous studies, which demonstrate that activated TGF- $\beta$ signaling in the tumor promotes T-cell exclusion, inhibits cytotoxic T-cell functions and provokes resistance to CBT. ${ }^{14-16}$ However, unlike in TNBC, where integrin $\alpha v \beta 6$-activated TGF- $\beta$ upregulates SOX4 in the tumor cells, causing resistance to $\mathrm{T}$ cell-mediated 

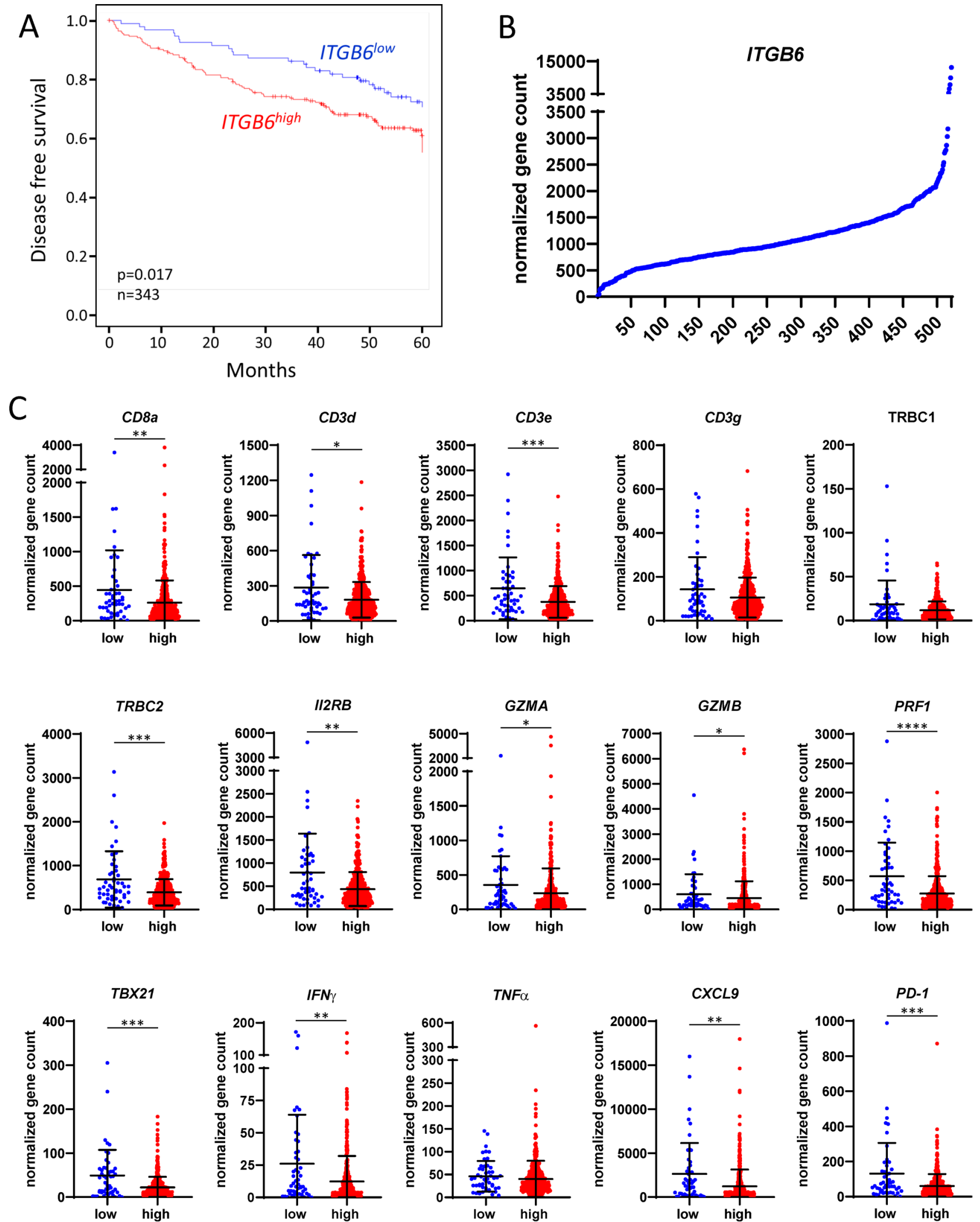

Figure 7 Integrin $\alpha v \beta 6$ inhibits T-cell immune response in the majority of patients with colorectal cancer. (A) Kaplan-Meier curves of disease free survival by ITGB6 gene expression status. Minimum p value approach was used to obtain an optimal discrimination of the total patient group into two subgroups with different disease-free survival depending on the level of ITGB6. Differences in survival were compared by logrank test. ITGB6 low: $n=109$, ITGB6 high: $n=234$. (B) Relative ITGB6 mRNA expression of all 521 patients in the TCGA-COAD dataset. (C) Relative mRNA expression in the 50 patients with lowest ITGB6 expression compared with the remaining 471 patients of the TCGA-COAD dataset. Means and SDs are shown. Mann-Whitney test was used to calculate statistical significance. ${ }^{*} \mathrm{P}<0.05,{ }^{* \star} \mathrm{p}<0.01,{ }^{* \star *} \mathrm{p}<0.001,{ }^{* \star \star *} \mathrm{p}<0.0001$. 
cytotoxicity, ${ }^{21}$ we did not detect an upregulation of SOX4 in our model, but identified a direct inhibiting effect of TGF- $\beta$ signaling on T-cells. In any respect, integrin $\alpha v \beta 6$ blockade is an efficient way to prevent the tumorpromoting effects of TGF- $\beta$ signaling in cancer.

Many efforts have been made to directly interfere in TGF- $\beta$ signaling with the intention to provoke a therapeutic response. However, since TGF- $\beta$ signaling is involved in a plethora of physiological processes, systemic TGF- $\beta$ blockade often leads to harmful off-target effects, resulting in limited success of TGF- $\beta$ inhibiting agents in clinical trials. ${ }^{17} 1820$ Therefore, blocking this pathway at the level of TGF- $\beta$ activation by inhibiting integrin $\alpha v \beta 6$ might be a much more promising and safe approach. Integrin $\alpha v \beta 6$ expression is restricted to epithelial cells and is mostly undetectable in tissue homeostasis of healthy epithelia. Apart from being upregulated during tumorigenesis of several epithelial cancers, it is only expressed during physiological events that require tissue remodeling, such as embryogenesis, endometrial cycle, wound healing, fibrosis and inflammation. ${ }^{1-3} 10$ This predominantly cancer-specific expression pattern presumably increases the therapeutic index of integrin $\alpha v \beta 6$ as a target for antibody blockade compared with the universally expressed TGF- $\beta$. Additionally, our data show that integrin $\alpha v \beta 6$ inhibits T-cells primarily locally in the tumor without causing systemic T-cell inhibition. Therefore, blockade of integrin $\alpha v \beta 6$ predominantly enhances T-cell activation in the tumor that expresses it and most likely does not cause systemic T-cell activation. In conclusion, our data demonstrate that integrin $\alpha v \beta 6$ represents a promising novel target for immunotherapy within the TGF- $\beta$ pathway.

In previous studies, integrin $\alpha v \beta 6$ inhibition has already been shown to be effective in preventing tumor growth and metastasis formation in vivo. ${ }^{6}{ }^{42}$ However, these studies did not discover the T-cell activating capacity of $\alpha v \beta 6$ inhibition and hypothesized, that other mechanisms are responsible for $\alpha v \beta 6$-induced cancer progression. They found that integrin $\alpha v \beta 6$ activates proliferation by enhancing ERK signaling, increases protease expression and enhances epithelial to mesenchymal transition (EMT)..${ }^{642}$ While these mechanisms might have an additional effect in promoting an invasive phenotype and enhancing tumor growth, our data suggest that the predominant tumor growth-promoting mechanism mediated by integrin $\alpha v \beta 6$ is T-cell exclusion and inhibition. Therefore, the correlation of integrin $\alpha v \beta 6$ expression with decreased survival in cancer might not only be caused by enhanced EMT and tumor invasion, but mainly by inhibition of the T-cell immune response.

There are two major activators of latent TGF- $\beta$ in vivo, integrin $\alpha v \beta 6$ and integrin $\alpha v \beta 8$. The particular importance of these two molecules for TGF- $\beta$ activation was demonstrated by inhibition of $\alpha v \beta 6$ in $\beta 8^{-/-}$mice, which reproduces the abnormalities of TGF- $\beta 1^{-/-}$mice. ${ }^{43}$ Both integrins activate TGF- $\beta$ by binding the RGD peptide in the LAP, thereby releasing active TGF- $\beta .^{11}{ }^{44}$ Integrin $\alpha v \beta 8$ has already been shown to enhance tumor growth by inhibiting infiltration of cytotoxic T-cells to the tumor. Furthermore, inhibition of $\alpha v \beta 8$ strongly enhances efficiency of $\alpha$ PD- 1 treatment and causes a potent inhibition of MC38 tumor growth. ${ }^{45}$ These data markedly resemble our findings with integrin $\alpha v \beta 6$ and highlight the importance of TGF- $\beta$ activation and signaling for the cytotoxic T-cell response. However, although integrin $\alpha v \beta 8$ was also found to be expressed in different epithelial malignancies, ${ }^{45}$ it also shows detectable protein expression levels in most healthy organs. ${ }^{46}$ Unlike integrin $\alpha v \beta 6$, which is primarily expressed in epithelial tumors, inhibition of $\alpha v \beta 8$ therefore poses a much higher risk for harmful offtarget effects. A recently published approach to combine $\alpha \mathrm{PD}-1$ treatment with an antibody that blocks both, integrin $\alpha v \beta 6$ and $\alpha v \beta 8^{21}$ might therefore largely eliminate physiological TGF- $\beta$ activation and lead to intolerable systemic side effects in patients, similar to direct TGF- $\beta$ inhibition.

CBT has become the standard of care for numerous malignancies. However, while CBT has shown to be especially effective to treat highly mutated tumors with numerous neoantigens, tumors with a low mutational burden show only limited response rates. ${ }^{47}$ In CRC, only patients that harbor microsatellite instability-high or DNA mismatch repair deficient (dMMR) tumors benefit from immunotherapy with PD-1 inhibitors and those patients represent only $3 \%-6 \%$ of all patients with advanced staged CRC. ${ }^{48}$ Therefore, improvement of immunotherapy for patients with microsatellite stable (MSS) or MMR-proficient (MMR-p) tumors is urgently needed. Up to this point, clinical studies aiming to enhance the immunogenic responses by combining PD-1 inhibitors with other treatment modalities in MSS or MMR-p disease were unsuccessful. ${ }^{48}$ Our data show that integrin $\alpha v \beta 6$ inhibition overcomes resistance to CBT, rendering the tumors susceptible to PD-1 inhibition. Therefore, a combination of CBT with inhibition of integrin $\alpha v \beta 6$ will potentially enhance an immunogenic response that might allow successful immunotherapy in MSS or MMR-p tumors. With regard to CRC, further studies will have to evaluate to what extent integrin $\alpha v \beta 6$ is responsible for CBT resistance of MSS or MMR-p tumors. However, since enhanced ITGB6 expression is observed in many cancers of epithelial origin, it is likely that $\alpha \mathrm{v} \beta 6$ upregulation is a general mechanism of immune response evasion and that combination of CBT with $\alpha v \beta 6$ inhibition will increase the response rates in multiple integrin $\alpha v \beta 6$ expressing cancer types.

Patients with CRC show varying levels of tumorous ITGB6 expression, which negatively correlate with disease free survival. Correspondingly, we showed that the majority of CRC tumors have sufficient ITGB6 expression to provoke inhibition of the cytotoxic T-cell response. Therefore, by treating these tumors with an ITGB6 blocking antibody, the antitumor T-cell response might be activated to a similar extent as in tumors with 
low ITGB6 expression and improve patient outcomes. In CRC, ITGB6 blockade therefore is a promising treatment strategy to enhance both, the T-cell response itself and the efficacy of other T-cell activating immunotherapies.

\section{Author affiliations}

${ }^{1}$ Department of Gastroenterology and Hepatology, University Hospital Zurich, University of Zurich, Zurich, Switzerland

${ }^{2}$ Division of Molecular and Experimental Surgery, Department of Surgery, FriedrichAlexander University Erlangen-Nuremberg, Erlangen, Germany

${ }^{3}$ Department of Physiology, University of Zurich, Zurich, Switzerland

${ }^{4}$ Institute of Pathology, University Hospital Erlangen, Friedrich-Alexander University Erlangen-Nuremberg, Erlangen, Germany

Acknowledgements We thank Paul Weinreb (Biogen) for the supply with antibodies. Further we thank Professor Lubor Borsig for providing us with CT26 and MC38-GFP cells. Additionally, we thank Katja Petter for excellent technical assistance.

Contributors Conceptualisation: PB, MS, MRS, GR, MSt. Formal analysis: PB, YM, EN. Investigation: PB, AM-A, EK, LH, KA, EN, CVP. Data curation: YM. Methodology: PB, SL, JFGG. Visualisation: PB, YM. Resources: AH. Writing - original draft preparation: PB. Writing - review and editing: all authors. Supervision: MS. Project administration: PB, MS. Funding acquisition: MS, MSt. Guarantor: MS

Funding The work was supported by a grant from the Stiftung Experimentelle Biomedizin to MS, a joint grant from the German Research Foundation/Swiss National Science Foundation to MS and MSt (320030E_190969), a grant from the Deutsche Forschungsgemeinschaft (DFG) - TRR 305, Subproject B08 to EN, a grant from the Swiss National Science Foundation to MS (320030_184753 and 314730_166381), a grant from the Cancer Research Center Zürich to MS and a grant from the Promedica Foundation to MS.

\section{Competing interests None declared.}

\section{Patient consent for publication Not applicable.}

Ethics approval All animal experiments were performed according to Swiss animal welfare legislation and approved by the local veterinary office (Veterinäramt des Kantons Zürich) (License numbers 239/2016 and 179/2019). Human sample collection and procedures were approved by the local ethics committee (no. 262_18 Bc; Ethikkommission der FAU, Erlangen, Germany). Informed consent was obtained from all patients before their participation in this study.

Provenance and peer review Not commissioned; externally peer reviewed.

Data availability statement RNA sequencing analysis data have been deposited in the BioProject database with accession number PRJNA798222.

Supplemental material This content has been supplied by the author(s). It has not been vetted by BMJ Publishing Group Limited (BMJ) and may not have been peer-reviewed. Any opinions or recommendations discussed are solely those of the author(s) and are not endorsed by BMJ. BMJ disclaims all liability and responsibility arising from any reliance placed on the content. Where the content includes any translated material, BMJ does not warrant the accuracy and reliability of the translations (including but not limited to local regulations, clinical guidelines, terminology, drug names and drug dosages), and is not responsible for any error and/or omissions arising from translation and adaptation or otherwise.

Open access This is an open access article distributed in accordance with the Creative Commons Attribution Non Commercial (CC BY-NC 4.0) license, which permits others to distribute, remix, adapt, build upon this work non-commercially, and license their derivative works on different terms, provided the original work is properly cited, appropriate credit is given, any changes made indicated, and the use is non-commercial. See http://creativecommons.org/licenses/by-nc/4.0/

\section{ORCID iD}

Philipp Busenhart http://orcid.org/0000-0002-5457-7927

\section{REFERENCES}

1 Bandyopadhyay A, Raghavan S. Defining the role of integrin alphavbeta6 in cancer. Curr Drug Targets 2009;10:645-52.

2 Niu J, Li Z. The roles of integrin $\alpha v \beta 6$ in cancer. Cancer Lett 2017;403:128-37.
3 Meecham A, Marshall JF. The ITGB6 gene: its role in experimental and clinical biology. Gene X 2020;5:100023.

4 Bates RC, Bellovin DI, Brown C, et al. Transcriptional activation of integrin beta6 during the epithelial-mesenchymal transition defines a novel prognostic indicator of aggressive colon carcinoma. J Clin Invest 2005;115:339-47.

5 Bengs $\mathrm{S}$, Becker $\mathrm{E}$, Busenhart $\mathrm{P}$, et al. $\beta_{6}$-integrin serves as a novel serum tumor marker for colorectal carcinoma. Int $J$ Cancer 2019;145:678-85.

6 Moore KM, Thomas GJ, Duffy SW, et al. Therapeutic targeting of integrin $\alpha v \beta 6$ in breast cancer. J National Cancer Institute 2014;106.

7 Reader CS, Vallath S, Steele CW, et al. The integrin $\alpha \mathrm{v} \beta 6$ drives pancreatic cancer through diverse mechanisms and represents an effective target for therapy. J Pathol 2019;249:332-42.

8 Elayadi AN, Samli KN, Prudkin L, et al. A peptide selected by biopanning identifies the integrin alphavbeta 6 as a prognostic biomarker for nonsmall cell lung cancer. Cancer Res 2007;67:5889-95.

9 Hazelbag S, Kenter GG, Gorter A, et al. Overexpression of the alpha $v$ beta 6 integrin in cervical squamous cell carcinoma is a prognostic factor for decreased survival. $J$ Pathol 2007;212:316-24.

10 Koivisto L, Bi J, Häkkinen L, et al. Integrin $\alpha v \beta 6$ : structure, function and role in health and disease. Int $J$ Biochem Cell Biol 2018;99:186-96.

11 Brown NF, Marshall JF. Integrin-Mediated TGF $\beta$ activation modulates the tumour microenvironment. Cancers2019;11:1221.

12 Batlle E, Massagué J. Transforming growth factor- $\beta$ signaling in immunity and cancer. Immunity 2019;50:924-40.

13 Dahmani A, Delisle J-S. TGF- $\beta$ in T cell biology: implications for cancer immunotherapy. Cancers 2018;10:1. doi:10.3390/ cancers10060194

14 Tauriello DVF, Palomo-Ponce S, Stork D, et al. TGF $\beta$ drives immune evasion in genetically reconstituted colon cancer metastasis. Nature 2018;554:538-43.

15 Mariathasan S, Turley SJ, Nickles D, et al. TGF $\beta$ attenuates tumour response to PD-L1 blockade by contributing to exclusion of T cells. Nature 2018;554:544-8.

16 Martin CJ, Datta A, Littlefield C, et al. Selective inhibition of TGF $\beta 1$ activation overcomes primary resistance to checkpoint blockade therapy by altering tumor immune landscape. Sci Trans/ Med 2020;12.

17 Akhurst RJ. Targeting TGF- $\beta$ signaling for therapeutic gain. Cold Spring Harb Perspect Biol 2017;9 doi:10.1101/cshperspect.a022301

18 Connolly EC, Freimuth J, Akhurst RJ. Complexities of TGF- $\beta$ targeted cancer therapy. Int J Biol Sci 2012;8:964-78.

19 Anderton MJ, Mellor HR, Bell A, et al. Induction of heart valve lesions by small-molecule ALK5 inhibitors. Toxicol Pathol 2011;39:916-24.

20 Huynh L, Hipolito C, ten Dijke P. A perspective on the development of TGF- $\beta$ inhibitors for cancer treatment. Biomolecules2019;9:743.

21 Bagati A, Kumar S, Jiang P, et al. Integrin $\alpha v \beta 6-T G F \beta-S O X 4$ pathway drives immune evasion in triple-negative breast cancer. Cancer Cell 2021;39:54-67.

22 Mohammed J, Beura LK, Bobr A, et al. Stromal cells control the epithelial residence of DCs and memory T cells by regulated activation of TGF- $\beta$. Nat Immunol 2016;17:414-21.

23 Campeau E, Ruhl VE, Rodier F, et al. A versatile viral system for expression and depletion of proteins in mammalian cells. PLoS One 2009;4:e6529.

24 Weinreb PH, Simon KJ, Rayhorn P, et al. Function-blocking integrin alphavbeta6 monoclonal antibodies: distinct ligand-mimetic and nonligand-mimetic classes. J Biol Chem 2004;279:17875-87.

25 Spalinger MR, Kasper S, Gottier C, et al. NLRP3 tyrosine phosphorylation is controlled by protein tyrosine phosphatase PTPN22. J Clin Invest 2016;126:1783-800.

26 Spalinger MR, Schwarzfischer M, Hering L, et al. Loss of PTPN22 abrogates the beneficial effect of cohousing-mediated fecal microbiota transfer in murine colitis. Mucosal Immunol 2019;12:1336-47.

27 Love Ml, Huber W, Anders S. Moderated estimation of fold change and dispersion for RNA-seq data with DESeq2. Genome Biol 2014; 15:550.

28 Huang DW, Sherman BT, Lempicki RA. Systematic and integrative analysis of large gene lists using DAVID bioinformatics resources. Nat Protoc 2009;4:44-57.

29 Huang DW, Sherman BT, Lempicki RA. Bioinformatics enrichment tools: paths toward the comprehensive functional analysis of large gene Lists. Nucleic Acids Res 2009;37:1-13.

30 Finotello F, Mayer C, Plattner C, et al. Molecular and pharmacological modulators of the tumor immune contexture revealed by deconvolution of RNA-seq data. Genome Med 2019;11:34. 
31 Robinson MD, McCarthy DJ, Smyth GK. edgeR: a Bioconductor package for differential expression analysis of digital gene expression data. Bioinformatics 2010;26:139-40.

32 McCarthy DJ, Chen Y, Smyth GK. Differential expression analysis of multifactor RNA-Seq experiments with respect to biological variation. Nucleic Acids Res 2012;40:4288-97.

33 Agrez M, Chen A, Cone Rl, et al. The alpha v beta 6 integrin promotes proliferation of colon carcinoma cells through a unique region of the beta 6 cytoplasmic domain. $J$ Cell Biol 1994;127:547-56.

34 Dixit RB, Chen A, Chen J, et al. Identification of a sequence within the integrin beta6 subunit cytoplasmic domain that is required to support the specific effect of alphavbeta6 on proliferation in threedimensional culture. J Biol Chem 1996;271:25976-80.

$35 \mathrm{Li}$ X, Yang Y, Hu Y, et al. Alphavbeta6-Fyn signaling promotes oral cancer progression. J Biol Chem 2003;278:41646-53.

36 Tokunaga R, Zhang W, Naseem M, et al. CXCL9, CXCL10, CXCL11/ CXCR3 axis for immune activation - A target for novel cancer therapy. Cancer Treat Rev 2018;63:40-7.

37 Calon A, Espinet E, Palomo-Ponce S, et al. Dependency of colorectal cancer on a TGF- $\beta$-driven program in stromal cells for metastasis initiation. Cancer Cell 2012;22:571-84.

38 Calon A, Lonardo E, Berenguer-Llergo A, et al. Stromal gene expression defines poor-prognosis subtypes in colorectal cancer. Nat Genet 2015;47:320-9.

39 Gorelik L, Flavell RA. Abrogation of TGFbeta signaling in T cells leads to spontaneous $T$ cell differentiation and autoimmune disease. Immunity 2000;12:171-81.
40 Vodenkova S, Buchler T, Cervena K, et al. 5-fluorouracil and other fluoropyrimidines in colorectal cancer: past, present and future. Pharmacol Ther 2020;206:107447.

41 Wu Y, Deng Z, Wang H, et al. Repeated cycles of 5-fluorouracil chemotherapy impaired anti-tumor functions of cytotoxic T cells in a CT26 tumor-bearing mouse model. BMC Immunol 2016;17:29.

42 Eberlein C, Kendrew J, McDaid K, et al. A human monoclonal antibody 264RAD targeting $\alpha v \beta 6$ integrin reduces tumour growth and metastasis, and modulates key biomarkers in vivo. Oncogene 2013;32:4406-16.

43 Aluwihare $\mathrm{P}, \mathrm{Mu} \mathrm{Z}$, Zhao Z, et al. Mice that lack activity of alphavbeta6- and alphavbeta8-integrins reproduce the abnormalities of Tgfb1- and Tgfb3-null mice. J Cell Sci 2009;122:227-32.

44 Shi M, Zhu J, Wang R, et al. Latent TGF- $\beta$ structure and activation. Nature 2011;474:343-9.

45 Takasaka N, Seed RI, Cormier A, et al. Integrin $\alpha v \beta 8$-expressing tumor cells evade host immunity by regulating TGF- $\beta$ activation in immune cells. JCl Insight 2018;3 doi:10.1172/jci.insight.122591

$46 \mathrm{McCarty} \mathrm{JH}$. $\alpha \mathrm{v} \beta 8$ integrin adhesion and signaling pathways in development, physiology and disease. J Cell Sci 2020;133 doi:10.1242/jcs.239434

47 Robert C. A decade of immune-checkpoint inhibitors in cancer therapy. Nat Commun 2020;11:3801.

48 Golshani G, Zhang Y. Advances in immunotherapy for colorectal cancer: a review. Therap Adv Gastroenterol 2020;13:1756284820917527. 